\title{
ALGEBRAIC-DELAY DIFFERENTIAL SYSTEMS, STATE-DEPENDENT DELAY, AND TEMPORAL ORDER OF REACTIONS
}

\author{
HANS-OTTO WALTHER
}

ABSTRACT. Systems of the form

$$
\begin{aligned}
x^{\prime}(t) & =g\left(r(t), x_{t}\right) \\
0 & =\Delta\left(r(t), x_{t}\right)
\end{aligned}
$$

generalize differential equations with delays $r(t)<0$ which are given implicitly by the history $x_{t}$ of the state. We show that the associated initial value problem generates a semiflow with differentiable solution operators on a Banach manifold. The theory covers reaction delays, signal transmission delays, threshold delays, and delays depending on the present state $x(t)$ only. As an application we consider a model for the regulation of the density of white blood cells and study monotonicity properties of the delayed argument function $\tau: t \mapsto t+r(t)$. There are solutions $(r, x)$ with $\tau^{\prime}(t)>0$ and others with $\tau^{\prime}(t)<0$. These other solutions correspond to feedback which reverses temporal order; they are short-lived and less abundant. Transient behaviour with a sign change of $\tau^{\prime}$ is impossible.

Key words: Delay differential equation, state-dependent delay, white blood cells, temporal order

AMS subject classification: 34 K 05, 37 L 99, 92 C 30 


\section{Introduction}

Consider an autonomous feedback system which reacts to its present state $x(t) \in \mathbb{R}$ after a delay which depends on the present state: The differential equation for this reads

$$
x^{\prime}(t+d(x(t)))=f(x(t)),
$$

with given functions $d: \mathbb{R} \rightarrow(0, \infty)$ and $f: \mathbb{R} \rightarrow \mathbb{R}$. It can be rewritten in the more familiar form

$$
x^{\prime}(s)=f(x(s+r(s)))
$$

with $s=t+d(x(t))$ and $r(s)=t-s<0$, which implies

$$
r(s)=t-s=-d(x(t))=-d(x(s+r(s))),
$$

hence

$$
0=d(x(t+r(t))+r(t) .
$$

In case of bounded delay $d(\mathbb{R}) \subset(0, h)$ for some $h>0$ we use the segment notation $x_{t}$ for the function $[-h, 0] \ni a \mapsto x(t+a) \in \mathbb{R}$, define $g=f \circ e v$ and $\Delta=d \circ e v+p r_{1}$ with the evaluation map

$$
e v:(-h, 0) \times \mathbb{R}^{[-h, 0]} \ni(s, \phi) \mapsto \phi(s) \in \mathbb{R}
$$

and the projection $p r_{1}$ onto the first component, and obtain the algebraic delay differential system

$$
\begin{aligned}
x^{\prime}(t) & =g\left(r(t), x_{t}\right) \\
0 & =\Delta\left(r(t), x_{t}\right) .
\end{aligned}
$$

The last equation defines the delay $r(t)$ implicitly by the segment $x_{t}$ of the state. As an example we shall discuss a model for the regulation of the density of white blood cells $[4,12]$. In the model the equation corresponding to $(1.1)$ is

$$
x^{\prime}(t+d(x(t)))=-\mu x(t+d(x(t)))+f(x(t)),
$$

with $\mu>0, d: \mathbb{R} \rightarrow(0, h)$ increasing and $f: \mathbb{R} \rightarrow \mathbb{R}$ decreasing. The associated system of the form (1.2)-(1.3) has $\Delta$ as before while $g=g_{\mu, f}$ is defined by

$$
\left.g_{\mu, f}(s, \phi)=-\mu \phi(0)+f(\phi(s))\right)=-\mu e v(0, \phi)+f \circ e v(s, \phi) .
$$

Several other differential equations with state-dependent delay can also be written in the form (1.2)-(1.3). We indicate how to define $\Delta$ in Eq. (1.3) for some of these cases.

(i) For delays $r(t)=-R\left(x_{t}\right)$ which are explicitly given by the state via a functional $R: \mathbb{R}^{[-h, 0]} \rightarrow(0, h)$, let

$$
\Delta(s, \phi)=s+R(\phi) .
$$

This includes delays $r(t)=\rho(x(t))$ which depend only on the present value of the state, via a function $\rho: \mathbb{R} \rightarrow(0, h)$, as in $[20,14,15,16,17,18,19,9,22]$.

(ii) For threshold delays $[1,10,11]$ (see also section 2.5 of the survey [6]) with a given continuous integral kernel $K: \mathbb{R}^{2} \rightarrow \mathbb{R}$ and a given threshold $\theta \in \mathbb{R}$ define $\Delta$ by

$$
\Delta(s, \phi)=\int_{s}^{0} K(\phi(0), \phi(u)) d u-\theta,
$$


for $-h<s<0$ and $\phi \in \mathbb{R}^{[-h, 0]}$ continuous.

(iii) For transmission delay as in $[25,26]$, with a given signal speed $c>0$ and a given lower bound $-w \leq 0$ for positions, consider

$$
\Delta(s, \phi)=c s+\phi(s)+\phi(0)+2 w .
$$

In the aforementioned work on threshold and transmission delays and also in [27], where the prototype system given by Eq. (1.1) is studied, hypotheses guarantee that Eq. (1.3) uniquely determines the delay $r(t)$ as a function of the state $x_{t}$. This reduces the algebraic delay differential system to a single delay differential equation. - Such hypotheses appear natural in cases of threshold and transmission delay but seem unrelated to modeling considerations for a reaction delay as in Eq. (1.1). Here a more general theory of the system (1.2)-(1.3) is desirable.

Before stating the main results of the present paper some notation is needed. For $h>0, n \in \mathbb{N}$, and $k \in \mathbb{N}$ we consider the Banach spaces $C=C\left([-h, 0], \mathbb{R}^{n}\right)$ with the norm given by

$$
\|\phi\|_{C}=\max _{-h \leq t \leq 0}|\phi(t)|
$$

$C^{1}=C^{1}\left([-h, 0], \mathbb{R}^{n}\right)$ with the norm given by

$$
\|\phi\|_{C^{1}}=\|\phi\|_{C}+\left\|\phi^{\prime}\right\|_{C},
$$

$B=\mathbb{R}^{k} \times C^{1}$ with the norm given by

$$
\|(s, \phi)\|_{B}=|s|+\|\phi\|_{C^{1}}
$$

and $\mathbb{R} \times B$ with the norm given by

$$
\|(t, s, \phi)\|_{\mathbb{R} \times B}=|t|+\|(s, \phi)\|_{B} .
$$

The space of continuous linear maps from a Banach space $\mathrm{X}$ into a Banach space $\mathrm{Y}$ is denoted by $L_{c}(X, Y)$.

For an open subset $U \subset B$ with $p r_{1} U \subset(-h, 0)^{k}$ we consider maps

$$
g: U \rightarrow \mathbb{R}^{n} \quad \text { and } \quad \Delta: U \rightarrow \mathbb{R}^{k} .
$$

A solution of the associated system (1.2)-(1.3) is a pair of functions $x:\left[t_{0}-h, t_{e}\right) \rightarrow$ $\mathbb{R}^{n}, r:\left[t_{0}, t_{e}\right) \rightarrow \mathbb{R}^{k}, t_{0}<t_{e} \leq \infty$, where $x$ is continuously differentiable, $r$ is continuous, $\left(r(t), x_{t}\right) \in U$ for all $t \in\left[t_{0}, t_{e}\right)$, Eq. (1.2) holds for all $t \in\left(t_{0}, t_{e}\right)$, and Eq. (1.3) holds for all $t \in\left[t_{0}, t_{e}\right)$. It is convenient to call the interval $\left[t_{0}, t_{e}\right)$ the domain of the solution. Solutions on intervals $\left(-\infty, t_{e}\right)$ with $t_{e} \leq \infty$ are defined with the appropriate modifications.

In the subsequent sections 1-7 the standing hypothesis $(\mathrm{H})$ is that

(H1) $g$ and $\Delta$ are continuously differentiable,

(H2) all derivatives $D g(s, \phi) \in L_{c}\left(B, \mathbb{R}^{n}\right)$ and $D_{2} \Delta(s, \phi) \in L_{c}\left(C^{1}, \mathbb{R}^{k}\right),(s, \phi) \in$ $U$, have linear extensions $D_{e} g(s, \phi): \mathbb{R}^{k} \times C \rightarrow \mathbb{R}^{n}$ and $D_{2, e} \Delta(s, \phi): C \rightarrow$ $\mathbb{R}^{k}$ 
(H3) both maps

$$
\Lambda: U \times \mathbb{R}^{k} \times C \ni(s, \phi, p, \chi) \mapsto D_{e} g(s, \phi)(p, \chi) \in \mathbb{R}^{n}
$$

and

$$
U \times C \ni(s, \phi, \chi) \mapsto D_{2, e} \Delta(s, \phi) \chi \in \mathbb{R}^{k}
$$

are continuous.

We prove that the set

$$
M=\left\{(s, \phi) \in U: \phi^{\prime}(0)=g(s, \phi), 0=\Delta(s, \phi), \operatorname{det} D_{1} \Delta(s, \phi) \neq 0\right\}
$$

is a continuously differentiable submanifold of codimension $k+n$ in $B$, and that the maximal solutions $\left(r^{s, \phi}, x^{s, \phi}\right)$ of the initial value problem given by the equations (1.2)-(1.3) for $t>0$ and

$$
\left(r(0), x_{0}\right)=(s, \phi)
$$

with $(s, \phi) \in M$ constitute a continuous semiflow $F:(t, s, \phi) \mapsto\left(r^{s, \phi}(t), x_{t}^{s, \phi}\right)$ on $M$. All solution operators $F_{t}=F(t, \cdot), t \geq 0$, are continuously differentiable. Their derivatives

$$
D F_{t}(s, \phi): T_{(s, \phi)} M \rightarrow T_{F(t, s, \phi)} M
$$

are given by linear variational equations. Moreover, $F$ is continuously differentiable for $t>h$.

The precise statements are contained in Propositions 3.1, 3.6, 4.3 and in Theorem 7.6 below. Hypothesis $(\mathrm{H})$ is a generalization of the hypotheses for the analogous results in $[23,24,6]$, for initial value problems of the form

$$
\begin{aligned}
x^{\prime}(t) & =\hat{f}\left(x_{t}\right) \\
x_{0} & =\phi \in X_{\hat{f}}=\left\{\phi \in \hat{U}: \phi^{\prime}(0)=\hat{f}(\phi)\right\},
\end{aligned}
$$

with $\hat{f}: C^{1} \supset \hat{U} \rightarrow \mathbb{R}^{n}$.

Let us mention here that further delay differential systems, where a bounded delay is defined implicitly by the state via a differential equation, like e. g. in $[2,13]$, are covered by the theory in $[23,24,6]$, under appropriate hypotheses on smoothness.

In section 8 we verify the properties $(\mathrm{H})$ for the white blood cell model in case $d: \mathbb{R} \rightarrow(0, h)$ and $f: \mathbb{R} \rightarrow \mathbb{R}$ are continuously differentiable. It is also easy to see that the functions $\Delta$ from the examples (i)-(iii) fulfill the relevant parts of hypothesis $(\mathrm{H})$ when restricted appropriately: In (i), consider the restriction of $R$ to $C^{1}$, assume that the latter is continuously differentiable, that derivatives have linear extensions $D_{e}\left(R \mid C^{1}\right)(\phi): C \rightarrow \mathbb{R}$, and that the map

$$
C^{1} \times C \ni(\chi, \phi) \mapsto D_{e}\left(R \mid C^{1}\right)(\phi) \chi \in \mathbb{R}
$$

is continuous. Consider $\Delta \mid(0, h) \times C^{1}$. If $\rho: \mathbb{R} \rightarrow(0, h)$ is continuously differentiable then $R$ given by $R(\phi)=\rho(\phi(0))$ fulfills the hypotheses just described. In (ii), assume that $K$ is continuously differentiable and consider $\Delta \mid(0, h) \times C^{1}$. In (iii), let

$$
h>\frac{2 w}{c} \text { and } U=(-h, 0) \times\left\{\phi \in C^{1}:-w<\phi(a)<\frac{c h}{2}-w \text { for }-h \leq a \leq 0\right\},
$$


and consider $\Delta \mid U$.

In order to find local solutions of the initial value problem (1.2)-(1.4) we employ the results from $[23,24,6]$, and avoid a new construction especially for the algebraicdelay differential system. In section 2 below the equation $0=\Delta(s, \phi)$ is solved by the Implicit Function Theorem for $s=\sigma_{0}(\phi)$ in a neighbourhood of an initial datum $\left(s_{0}, \phi_{0}\right)$ which satisfies $\Delta\left(s_{0}, \phi_{0}\right)=0$, det $D_{1} \Delta\left(s_{0}, \phi_{0}\right) \neq 0$, and $\left(\phi_{0}\right)^{\prime}(0)=g\left(s_{0}, \phi_{0}\right)$. Upon that the theory of $[23,24,6]$ is applied to the initial value problem

$$
\begin{aligned}
& x^{\prime}(t)=g\left(\sigma_{0}\left(x_{t}\right), x_{t}\right), \\
& x_{0}=\phi
\end{aligned}
$$

with $\phi^{\prime}(0)=g\left(\sigma_{0}(\phi), \phi\right)$. Section 3 begins with the proof that the set $M$ is a continuously differentiable submanifold of the space $B$. For initial data in $M$ the result of section 2 yields local solutions $(r, x)$ of the problem (1.2)-(1.4), with $r(t)=\sigma_{0}\left(x_{t}\right)$ (Proposition 3.3). Then we proceed to maximal solutions, obtain the semiflow $F$ on $M$ and prove that $F$ is continuous. Also, we find a differential equation (*) for the $r$-component of solutions (Proposition 3.4). - This may suggest to replace Eq. (1.3) with Eq. $(*)$ and to study the new system in the framework of $[23,24,6]$. It should however be noticed that the map on the right hand side of Eq. (*) in general fails to satisfy the hypotheses needed in $[23,24,6]$. - Having the semiflow $F$ we formulate its relation to the semiflows from section 2 in terms of flow equivalence (Proposition 3.7). This should be convenient for the transfer of results, notably on local invariant manifolds, which have been obtained for initial value problems of the form (1.5)-(1.6) $[6,8,21]$.

The remaining smoothness properties of the semiflow $F$ are proved in sections 4-7. We follow the approach in $[23,24]$ as closely as possible. Proposition 3.4 is used in section 5 on the derivative $D_{1} F(t, s, \phi)$, which exists for $t>h$.

In the final section 8 of this paper we discuss the white blood cell model as an example for feedback with a reaction delay, in the sense explained at the beginning in the context of Eq. (1.1). Now the $r$-components of solutions are scalar, and one may ask whether for a given solution $(r, x)$ the associated delayed argument function

$$
\tau: t \mapsto t+r(t)
$$

(which in the example appears on the right hand side of Eq. (1.2)) is increasing or not.

In many contributions on differential equations with state-dependent delay the delayed argument functions are increasing, see the survey paper [6]. This property may be felt natural and seems to facilitate the analysis.

A decrease of $\tau$, on the other hand, means that the system reacts to states $\xi_{1}=$ $x\left(\tau\left(t_{1}\right)\right), \xi_{2}=x\left(\tau\left(t_{2}\right)\right)$ in the past, with $\tau\left(t_{1}\right)<\tau\left(t_{2}\right)$, in reverse temporal order, namely by reactions $x^{\prime}\left(t_{1}\right)$ to $\xi_{1}$ and $x^{\prime}\left(t_{2}\right)$ to $\xi_{2}$ at $t_{2}<t_{1}$.

In the experiment by Libet et al. [7] on unconscious brain activity before the moment of awareness and voluntary action it was found that awareness of certain 
external stimuli in short time intervals arises in reverse temporal order. This may be taken as an indication that decreasing delayed argument functions have a counterpart in biological reality.

Recall that a flowline of the semiflow $F$ is a map $I \ni t \mapsto(r(t), X(t)) \in M$, with $I \subset$ $\mathbb{R}$ an interval, so that for all $t<u$ in $I$ we have $(r(u), X(u))=F(u-t, r(t), X(t))$.

In our example the manifold $M$ decomposes into an open subset $M_{+} \neq \emptyset$ formed by flowlines with strictly increasing delayed argument function, and into another open subset $M_{-} \neq \emptyset$ formed by flowlines with strictly decreasing delayed argument function (Proposition 8.2). The flowlines in $M_{-}$are short-lived, for the trivial reason that $\tau(t) \geq t-h$ (Corollary 8.3).

In case $f$ is bounded each flowline $F(\cdot, s, \phi)$ with bounded domain $\left[0, t_{s, \phi}\right), t_{s, \phi}<\infty$, converges to a limit point in the manifold boundary $\bar{M} \backslash M$ as $t \nearrow t_{s, \phi}$ (Proposition 8.5). Unless $d$ is constant there exist points in $\bar{M} \backslash M$ from which pairs of flowlines bifurcate, one into the component $M_{+}$and the other one into $M_{-}$(Proposition 8.7). Also, certain flowlines in $M_{+}$and in $M_{-}$terminate at limit points with the same first (delay) component (Proposition 8.8).

One may ask whether there exists continuous transient behaviour in the sense that a flowline in $M_{+}$terminates at a point in the manifold boundary and can be continued from there by a flowline in $M_{-}$, or vice versa. Proposition 8.6 says that such behaviour is impossible.

Notation, preliminaries. For derivatives of functions $x: J \rightarrow E, J \subset \mathbb{R}$ and $E$ a Banach space over $\mathbb{R}$, we have $D x(t) \in L_{c}(\mathbb{R}, E)$ and $x^{\prime}(t)=D x(t) 1 \in E$.

The evaluation map $e v_{0}=e v \mid(-h, 0) \times C$ (with values in $\left.\mathbb{R}^{n}\right)$ is continuous but not locally Lipschitz continuous. The restriction $e v_{1}=e v \mid(-h, 0) \times C^{1}$ (values in $\mathbb{R}^{n}$ ) is continuously differentiable with

$$
D e v_{1}(s, \phi)(\hat{s}, \hat{\phi})=D_{1} e v_{1}(s, \phi) \hat{s}+D_{2} e v_{1}(s, \phi) \hat{\phi}=\hat{s} \phi^{\prime}(s)+\hat{\phi}(s) .
$$

We also need the linear continuous maps

$$
e v_{0, s}: C \ni \chi \mapsto \chi(s) \in \mathbb{R}^{n} \quad \text { and } \quad e v_{1, s}: C^{1} \ni \phi \mapsto \phi(s) \in \mathbb{R}^{n},
$$

for $s \in[-h, 0]$, and

$$
\partial: C^{1} \ni \phi \mapsto \phi^{\prime} \in C .
$$

For a given Cartesian product of sets the projection onto the $j$-th component is always denoted by $p r_{j}$. 


\section{Restricted initial VAlue PROBlems}

We begin with a local solution of the equation

$$
0=\Delta(s, \phi)
$$

Let $\left(s_{0}, \phi_{0}\right) \in U$ with

$$
0=\Delta\left(s_{0}, \phi_{0}\right)
$$

be given and assume det $D_{1} \Delta\left(s_{0}, \phi_{0}\right) \neq 0$. We apply the Implicit Function Theorem and obtain open neighbourhoods $V_{0} \subset(-h, 0)^{k}$ of $s_{0}, N_{0} \subset C^{1}$ of $\phi_{0}$ with $V_{0} \times N_{0} \subset$ $U$ and

$$
0 \neq \operatorname{det} D_{1} \Delta(s, \phi) \quad \text { on } \quad V_{0} \times N_{0},
$$

and a continuously differentiable map $\sigma_{0}: N_{0} \rightarrow V_{0}$ with

$$
\begin{aligned}
\sigma_{0}\left(\phi_{0}\right) & =s_{0}, \\
0 & =\Delta\left(\sigma_{0}(\phi), \phi\right) \text { on } N_{0}, \\
\left(V_{0} \times N_{0}\right) \cap \Delta^{-1}(0) & =\left(\sigma_{0} \times \operatorname{id}_{N_{0}}\right)\left(N_{0}\right) .
\end{aligned}
$$

We look for solutions of the restricted initial value problem

$$
\begin{aligned}
x^{\prime}(t) & =g_{\sigma_{0}}\left(x_{t}\right), \\
x_{0} & =\phi,
\end{aligned}
$$

with the functional $g_{\sigma_{0}}: N_{0} \rightarrow \mathbb{R}^{n}$ given by

$$
g_{\sigma_{0}}(\phi)=g\left(\sigma_{0}(\phi), \phi\right)
$$

A solution is a continuously differentiable function $x:\left[-h, t_{e}\right) \rightarrow \mathbb{R}^{n}, 0<t_{e} \leq \infty$, with $x_{t} \in N_{0}$ on $\left[0, t_{e}\right)$ and $x_{0}=\phi$ which satisfies Eq. (2.4) for $0<t<t_{e}$.

In order to apply the results from $[23,24,6]$ on existence, uniqueness and smoothness we have to verify the hypothesis $\left(\mathrm{H}, \sigma_{0}\right)$ that

$\left(\mathrm{H} 1, \sigma_{0}\right) g_{\sigma_{0}}$ is continuously differentiable,

$\left(\mathrm{H} 2, \sigma_{0}\right)$ each derivative $D g_{\sigma_{0}}(\phi) \in L_{c}\left(C^{1}, \mathbb{R}^{n}\right)$ has an extension $D_{e} g_{\sigma_{0}}(\phi)$ in $L_{c}\left(C, \mathbb{R}^{n}\right)$,

(H3, $\left.\sigma_{0}\right)$ the map $N_{0} \times C \ni(\phi, \chi) \mapsto D_{e} g_{\sigma_{0}}(\phi) \chi \in \mathbb{R}^{n}$ is continuous.

We begin with the derivatives of $\sigma_{0}$. From

$$
\begin{aligned}
0 & =D\left(\Delta \circ\left(\sigma_{0} \times i d\right)\right)(\phi) \chi \\
& =D_{1} \Delta\left(\sigma_{0}(\phi), \phi\right) D \sigma_{0}(\phi) \chi+D_{2} \Delta\left(\sigma_{0}(\phi), \phi\right) \chi
\end{aligned}
$$

for $\phi \in N_{0}$ and $\chi \in C^{1}$ we get

$$
D \sigma_{0}(\phi) \chi=-\left(D_{1} \Delta\left(\sigma_{0}(\phi), \phi\right)\right)^{-1}\left(D_{2} \Delta\left(\sigma_{0}(\phi), \phi\right) \chi\right)
$$

and see that each derivative $D \sigma_{0}(\phi) \in L_{c}\left(C^{1}, \mathbb{R}^{k}\right), \phi \in N_{0}$, has a continuous linear extension $D_{e} \sigma_{0}(\phi): C \rightarrow \mathbb{R}^{k}$, which is given by the same formula as $D \sigma_{0}(\phi)$, namely,

$$
D_{e} \sigma_{0}(\phi) \chi=-\left(D_{1} \Delta\left(\sigma_{0}(\phi), \phi\right)\right)^{-1}\left(D_{2, e} \Delta\left(\sigma_{0}(\phi), \phi\right) \chi\right) .
$$

Hence the map

$$
N_{0} \times C \ni(\phi, \chi) \mapsto D_{e} \sigma_{0}(\phi) \chi \in \mathbb{R}^{k}
$$


is continuous (with respect to the product topology given by the norms on $C^{1}$ and on $C$ ). For $\phi \in N_{0}$ and $\chi \in C^{1}$ we have

$$
D g_{\sigma_{0}}(\phi) \chi=D g\left(\sigma_{0}(\phi), \phi\right)\left(D \sigma_{0}(\phi) \chi, \chi\right)
$$

Using the continuous linear extensions $D_{e} g\left(\sigma_{0}(\phi), \phi\right): \mathbb{R}^{k} \times C \rightarrow \mathbb{R}^{n}$ and $D_{e} \sigma_{0}(\phi)$ : $C \rightarrow \mathbb{R}^{k}$ we infer that also each map $D g_{\sigma_{0}}(\phi), \phi \in N_{0}$, has a continuous linear extension $D_{e} g_{\sigma_{0}}(\phi): C \rightarrow \mathbb{R}^{n}$ given by

$$
D_{e} g_{\sigma_{0}}(\phi) \chi=D_{e} g\left(\sigma_{0}(\phi), \phi\right)\left(D_{e} \sigma_{0}(\phi) \chi, \chi\right)
$$

and that $\left(\mathrm{H} 3, \sigma_{0}\right)$ holds.

Let

$$
X_{\sigma_{0}}=\left\{\phi \in N_{0}: \phi^{\prime}(0)=g_{\sigma_{0}}(\phi)\right\} .
$$

From $[23,24,6]$ we now have the following result.

Proposition 2.1. Let $\left(s_{0}, \phi_{0}\right) \in U$ be given with

$$
0=\Delta\left(s_{0}, \phi_{0}\right) \quad \text { and } \quad 0 \neq \operatorname{det} D_{1} \Delta\left(s_{0}, \phi_{0}\right) .
$$

Then there exist open neighbourhoods $V_{0} \subset(-h, 0)^{k}$ of $s_{0}$ and $N_{0} \subset C^{1}$ of $\phi_{0}$ with $V_{0} \times N_{0} \subset U$ so that

$$
\operatorname{det} D_{1} \Delta(s, \phi) \neq 0 \quad \text { on } \quad V_{0} \times N_{0} \text {, }
$$

and there is a continuously differentiable map $\sigma_{0}: N_{0} \rightarrow V_{0}$ with properties (2.1)(2.3) so that the following holds.

(i) The set $X_{\sigma_{0}} \subset N_{0}$ is a continuously differentiable submanifold of $C^{1}$ with codimension $n$, and for each $\phi \in X_{\sigma_{0}}$ we have

$$
T_{\phi} X_{\sigma_{0}}=\left\{\chi \in C^{1}: \chi^{\prime}(0)=D g_{\sigma_{0}}(\phi) \chi\right\} .
$$

(ii) Each $\phi \in X_{\sigma_{0}}$ defines a unique maximal solution $x^{\sigma_{0}, \phi}:\left[-h, t_{\sigma_{0}, \phi}\right) \rightarrow \mathbb{R}^{n}$, $0<t_{\sigma_{0}, \phi} \leq \infty$, of the initial value problem (2.4)-(2.5). We have $x_{t}^{\sigma_{0}, \phi} \in X_{\sigma_{0}}$ for all $t \in\left[0, t_{\sigma_{0}, \phi}\right)$, and the equation

$$
F_{\sigma_{0}}(t, \phi)=x_{t}^{\sigma_{0}, \phi}
$$

defines a continuous semiflow

$$
F_{\sigma_{0}}: \Omega_{\sigma_{0}} \rightarrow X_{\sigma_{0}}, \quad \Omega_{\sigma_{0}}=\left\{(t, \phi) \in[0, \infty) \times X_{\sigma_{0}}: 0 \leq t<t_{\sigma_{0}, \phi}\right\} .
$$

(iii) Each map

$$
F_{\sigma_{0}, t}: \Omega_{\sigma_{0}, t} \ni \phi \mapsto F_{\sigma_{0}}(t, \phi) \in X_{\sigma_{0}}
$$

with $t \geq 0$ and $\Omega_{\sigma_{0}, t}=\left\{\phi \in X_{\sigma_{0}}: t<t_{\sigma_{0}, \phi}\right\} \neq \emptyset$ is continuously differentiable. For $\phi \in \Omega_{\sigma_{0}, t}, x=x^{\sigma_{0}, \phi}$, and $r:\left[0, t_{\sigma_{0}, \phi}\right) \rightarrow \mathbb{R}^{k}$ given by $r(t)=\sigma_{0}\left(x_{t}\right)$ we have

$$
0 \neq \operatorname{det} D_{1} \Delta\left(r(u), x_{u}\right) \quad \text { for } \quad 0 \leq u<t_{\sigma_{0}, \phi}
$$

and

$$
D F_{\sigma_{0}, t}(\phi) \chi=v_{t}
$$

with $v:\left[-h, t_{\sigma_{0}, \phi}\right) \rightarrow \mathbb{R}^{n}$ continuously differentiable,

$$
\begin{aligned}
v^{\prime}(u) & =D g_{\sigma_{0}}\left(x_{u}\right) v_{u} \text { for } 0<u<t_{\sigma_{0}, \phi}, \\
v_{0} & =\chi .
\end{aligned}
$$


(iv) The restriction of $F_{\sigma_{0}}$ to the open subset

$$
\left\{(t, \phi) \in \Omega_{\sigma_{0}}: t>h\right\}
$$

of the submanifold $\mathbb{R} \times X_{\sigma_{0}}$ of the Banach space $\mathbb{R} \times C^{1}$ is continuously differentiable, with

$$
D_{1} F_{\sigma_{0}}(t, \phi) 1=\left(x^{\sigma_{0}, \phi}\right)_{t}^{\prime}
$$




\section{The SEMiflow On A MANifold in $B$}

Now we address the initial value problem (1.2)-(1.4). We assume that hypothesis $(\mathrm{H})$ holds, and look for maximal solutions with initial data in $(s, \phi) \in M \subset$ $\mathbb{R}^{k} \times C^{1}=B$. The idea is to continue local solutions by means of the Implicit Function Theorem and Proposition 2.1 as long as $0 \neq \operatorname{det} D_{1} \Delta\left(r(t), x_{t}\right)$.

Proposition 3.1. The set

$$
M=\left\{(s, \phi) \in U: \phi^{\prime}(0)=g(s, \phi), \Delta(s, \phi)=0, \text { det } D_{1} \Delta(s, \phi) \neq 0\right\}
$$

is a continuously differentiable submanifold of $B$, with codimension $k+n$.

Proof. Let $\mathcal{O}=\left\{(s, \phi) \in U: \operatorname{det} D_{1} \Delta(s, \phi) \neq 0\right\}$. $M$ is the zeroset of the continuously differentiable map $Z: \mathcal{O} \rightarrow \mathbb{R}^{k} \times \mathbb{R}^{n}$ with the components $Z_{1}=\Delta \mid \mathcal{O}$ and

$$
Z_{2}=e v_{0,0} \circ \partial \circ p r_{2}|\mathcal{O}-g| \mathcal{O} \text {. }
$$

It is enough to show that for every $(s, \phi) \in M$ the derivative $D Z(s, \phi)$ is surjective. (Then $\mathrm{B}$ has a direct sum decomposition into the closed nullspace $Y=$ $D Z(s, \phi)^{-1}(0)$ and a complementary subspace $Q$ of dimension $k+n$, and the Implicit Function Theorem shows that locally the translate $M-(s, \phi)$ is given by a continuously differentiable map $\gamma: V \rightarrow Q, V$ an open neighbourhood of 0 , with $\gamma(0)=0$ and $D \gamma(0)=0$.) Let $(s, \phi) \in M$. Then

$$
D Z_{1}(s, \phi)(p, \chi)=D_{1} \Delta(s, \phi) p+D_{2} \Delta(s, \phi) \chi=D_{1} \Delta(s, \phi) p+D_{2, e} \Delta(s, \phi) \chi
$$

and

$$
D Z_{2}(s, \phi)(p, \chi)=\chi^{\prime}(0)-D g(s, \phi)(p, \chi)=\chi^{\prime}(0)-D_{e} g(s, \phi)(p, \chi)
$$

for all $(p, \chi) \in B$. We look for a basis of $\mathbb{R}^{k} \times \mathbb{R}^{n}$ in $D Z(s, \phi) B$. Let $e_{1}, \ldots, e_{k}$ denote the canonical basis of $\mathbb{R}^{k}$, and let $e_{k+1}, \ldots, e_{k+n}$ denote the canonical basis of $\mathbb{R}^{n}$. As $D_{1} \Delta(s, \phi): \mathbb{R}^{k} \rightarrow \mathbb{R}^{k}$ is an isomorphism there exist $p_{1}, \ldots, p_{k}$ in $\mathbb{R}^{k}$ and $a_{1}, \ldots, a_{k} \in \mathbb{R}^{n}$ so that

$$
\begin{aligned}
D Z(s, \phi)\left(p_{j}, 0\right) & =\left(\begin{array}{c}
D Z_{1}(s, \phi)\left(p_{j}, 0\right) \\
D Z_{2}(s, \phi)\left(p_{j}, 0\right)
\end{array}\right) \\
& =\left(\begin{array}{c}
D D_{1} \Delta(s, \phi) p_{j} \\
D Z_{2}(s, \phi)\left(p_{j}, 0\right)
\end{array}\right)=\left(\begin{array}{c}
e_{j} \\
a_{j}
\end{array}\right) \in \mathbb{R}^{k} \times \mathbb{R}^{n}
\end{aligned}
$$

for $j=1, \ldots, k$. Choose $n$ sequences $\left(\chi_{j}^{(\nu}\right)_{\nu=1}^{\infty}$ in $C^{1}, j \in\{k+1, \ldots, k+n\}$, so that

$$
\begin{aligned}
\left(\chi_{j}^{(\nu)}\right)^{\prime}(0) & =e_{j} \text { for all } j \in\{k+1, \ldots, k+n\} \quad \text { and } \nu \in \mathbb{N}, \\
0 & =\lim _{\nu \rightarrow \infty}\left\|\chi_{j}^{(\nu)}\right\|_{C} \text { for all } j \in\{k+1, \ldots, k+n\} .
\end{aligned}
$$

By continuity of $D_{2, e} \Delta(s, \phi)$ and $D_{e} g(s, \phi)$,

$$
D Z(s, \phi)\left(0, \chi_{j}^{(\nu)}\right)=\left(\begin{array}{c}
D_{2, e} \Delta(s, \phi) \chi_{j}^{(\nu)} \\
\left(\chi_{j}^{(\nu)}\right)^{\prime}(0)-D_{e} g(s, \phi)\left(0, \chi_{j}^{(\nu)}\right)
\end{array}\right) \rightarrow\left(\begin{array}{c}
0 \\
e_{j}
\end{array}\right) \quad \text { as } \quad \nu \rightarrow \infty
$$

for each $j \in\{k+1, \ldots, k+n\}$. It follows that for $\nu \rightarrow \infty$ the determinants of the matrices

$$
\left(D Z(s, \phi)\left(p_{1}, 0\right), \ldots, D Z(s, \phi)\left(p_{k}, 0\right), D Z(s, \phi)\left(0, \chi_{k+1}^{(\nu)}\right), \ldots, D Z(s, \phi)\left(0, \chi_{k+n}^{(\nu)}\right)\right)
$$

converge to 1 , which implies that $D Z(s, \phi) B$ contains a basis of $\mathbb{R}^{k} \times \mathbb{R}^{n}$. 
For $(s, \phi) \in M$ the tangent space $T_{(s, \phi)} M$ coincides with the nullspace $D Z(s, \phi)^{-1}(0)$ from the preceding proof. Hence

$$
T_{(s, \phi)} M=\left\{(p, \chi) \in B: 0=D \Delta(s, \phi)(p, \chi), \quad \chi^{\prime}(0)=D g(s, \phi)(p, \chi)\right\} .
$$

It is convenient to introduce another description of the tangent space, in terms of the map

$$
A_{1}: M \ni(s, \phi) \mapsto-\left(D_{1} \Delta(s, \phi)\right)^{-1} \circ D_{2} \Delta(s, \phi) \in L_{c}\left(C^{1}, \mathbb{R}^{k}\right) .
$$

Corollary 3.2. For $(s, \phi) \in M$,

$$
T_{(s, \phi)} M=\left\{(p, \chi) \in B: p=A_{1}(s, \phi) \chi, \quad \chi^{\prime}(0)=D g(s, \phi)\left(A_{1}(s, \phi) \chi, \chi\right)\right\} .
$$

Proof. Use that the equation $0=D \Delta(s, \phi)(p, \chi)=D_{1} \Delta(s, \phi) p+D_{2} \Delta(s, \phi) \chi$ is equivalent to $p=-\left(D_{1} \Delta(s, \phi)^{-1}\left(D_{2} \Delta(s, \phi) \chi\right)\right.$, as $D_{1} \Delta(s, \phi)$ is an isomorphism.

Notice that the map $A_{1}$ is continuous. Later we shall also use the continuous map

$$
A: M \times C \ni(s, \phi, \chi) \mapsto-\left(D_{1} \Delta(s, \phi)\right)^{-1}\left(D_{2, e} \Delta(s, \phi) \chi\right) \in \mathbb{R}^{k} .
$$

For $(s, \phi) \in M$ and $\chi \in C^{1}$ we have

$$
A(s, \phi, \chi)=A_{1}(s, \phi) \chi .
$$

Proposition 3.3. (Existence of solutions to the initial value problem) Let $\left(s_{0}, \phi_{0}\right) \in$ $M$ be given. Then (2.7) holds, and there are $V_{0}, N_{0}, \sigma_{0}, X_{\sigma_{0}}$ as in Proposition 2.1. There exist a further open neighbourhood $N_{01} \subset N_{0}$ of $\phi_{0}$ in $C^{1}$ and $t_{0}>0$ so that for every $(s, \phi) \in M \cap\left(V_{0} \times N_{01}\right)$ we have $s=\sigma_{0}(\phi), \phi \in X_{\sigma_{0}}, t_{0}<t_{\sigma_{0}, \phi}$, $x_{t}^{\sigma_{0}, \phi} \in N_{0}$ for $0 \leq t \leq t_{0}$, and the equations $r(t)=\sigma_{0}\left(x_{t}^{\sigma_{0}, \phi}\right)$ and $x(t)=x^{\sigma_{0}, \phi}(t)$ define a solution $(r, x)$ of the initial value problem (1.2)-(1.4) with domain $\left[0, t_{0}\right)$, and with $\left(r(t), x_{t}\right) \in M$ on $\left[0, t_{0}\right)$.

Proof. The domain $\Omega_{\sigma_{0}}$ is open in $[0, \infty) \times X_{\sigma_{0}}$. Using this and continuity of the semiflow $F_{\sigma_{0}}$ at $\left(0, \phi_{0}\right) \in \Omega_{\sigma_{0}} \subset[0, \infty) \times X_{\sigma_{0}}$ we obtain an open neighbourhood $N_{01}$ of $\phi_{0}$ in $N_{0}$ and $t_{0}>0$ with $\left[0, t_{0}\right] \times\left(X_{\sigma_{0}} \cap N_{01}\right) \subset \Omega_{\sigma_{0}}$ and $F_{\sigma_{0}}\left(\left[0, t_{0}\right] \times N_{01}\right) \subset N_{0}$. Let $(s, \phi) \in M \cap\left(V_{0} \times N_{01}\right)$ be given. Then $\Delta(s, \phi)=0$ and $(s, \phi) \in V_{0} \times N_{0}$, hence $s=\sigma_{0}(\phi)$. Also,

$$
\phi^{\prime}(0)=g(s, \phi)=g\left(\sigma_{0}(\phi), \phi\right)=g_{\sigma_{0}}(\phi),
$$

or $\phi \in X_{\sigma_{0}}$. Let $\xi=x^{\sigma_{0}, \phi}$. We infer $\xi_{t}=x_{t}^{\sigma_{0}, \phi} \in N_{0}$ for $0 \leq t \leq t_{0}$. Consider the continuous function $r:\left[0, t_{0}\right] \ni t \mapsto \sigma_{0}\left(\xi_{t}\right) \in \mathbb{R}^{k}$ and the continuously differentiable function $x:\left[-h, t_{0}\right) \ni t \mapsto \xi(t) \in \mathbb{R}^{n}$. We obtain $\Delta\left(r(t), x_{t}\right)=\Delta\left(\sigma_{0}\left(\xi_{t}\right), \xi_{t}\right)=0$ for $0 \leq t \leq t_{0}$ and

$$
x^{\prime}(t)=\xi^{\prime}(t)=g_{\sigma_{0}}\left(\xi_{t}\right)=g\left(\sigma_{0}\left(\xi_{t}\right), \xi_{t}\right)=g\left(r(t), x_{t}\right)
$$

for $0<t<t_{0}$. Also, $r(0)=\sigma_{0}\left(\xi_{0}\right)=\sigma_{0}(\phi)=s, x_{0}=\xi_{0}=\phi$. In particular,

$$
x^{\prime}(0)=\phi^{\prime}(0)=g(s, \phi)=g\left(r(0), x_{0}\right) \text {. }
$$

Finally, for $0 \leq t \leq t_{0}$ we have $\operatorname{det} D_{1} \Delta\left(r(t), x_{t}\right)=\operatorname{det} D_{1} \Delta\left(\sigma_{0}\left(\xi_{t}\right), \xi_{t}\right) \neq 0$, due to $(2.7)$ and $\left(\sigma_{0}\left(\xi_{t}\right), \xi_{t}\right) \in V_{0} \times N_{0}$. Altogether it follows that $\left(r(t), x_{t}\right) \in M$ on $\left[0, t_{0}\right]$. 
Proposition 3.4. For any solution $(r, x)$ of the initial value problem (1.2)-(1.4) with $(s, \phi) \in M$ we have

$$
0 \neq \operatorname{det} D_{1} \Delta\left(r(t), x_{t}\right) \quad \text { for } \quad 0 \leq t<t_{e}
$$

and the map $r$ is continuously differentiable with

$$
r^{\prime}(t)=A\left(r(t), x_{t}, \partial x_{t}\right)
$$

for all $t \in\left[0, t_{e}\right)$.

Proof. 1. The inequality holds as $\left(r(t), x_{t}\right) \in M$ for $0 \leq t<t_{e}$.

2. The set

$$
S_{x}=\left\{(s, t) \in(-h, 0)^{k} \times\left(0, t_{e}\right):\left(s, x_{t}\right) \in U\right\}
$$

is open since the map $\left[0, t_{e}\right) \ni t \mapsto x_{t} \in C^{1}$ is continuous and $U$ is open. For all $t \in\left(0, t_{e}\right),\left(r(t), x_{t}\right) \in S_{x}$. The map

$$
\Delta_{x}: S_{x} \ni(s, t) \mapsto \Delta\left(s, x_{t}\right) \in \mathbb{R}^{k}
$$

has partial derivatives $D_{1} \Delta_{x}(s, t)=D_{1} \Delta\left(s, x_{t}\right)$ at each $(s, t) \in S_{x}$, and $D_{1} \Delta_{x}$ : $S_{x} \rightarrow L_{c}\left(\mathbb{R}^{k}, \mathbb{R}^{k}\right)$ is continuous.

3. We show that at each $(s, t) \in S_{x}$ the partial derivative $D_{2} \Delta_{x}(s, t) \in L_{c}\left(\mathbb{R}, \mathbb{R}^{k}\right)$ exists and is given by

$$
D_{2} \Delta_{x}(s, t) 1=D_{2, e} \Delta\left(s, x_{t}\right) \partial x_{t} .
$$

Let $(s, t) \in S_{x}$ be given. There exists $\epsilon>0$ with $0<t-\epsilon, t+\epsilon<t_{e}$ such that for all $\tau \in(-\epsilon, \epsilon)$ and for all $\theta \in[0,1]$ we have $\left(s, x_{t}+\theta\left(x_{t+\tau}-x_{t}\right)\right) \in U$. In case $\tau \neq 0$ we get

$$
\begin{gathered}
\Delta_{x}(s, t+\tau)-\Delta_{x}(s, t)-\tau D_{2, e} \Delta\left(s, x_{t}\right) \partial x_{t}=\Delta\left(s, x_{t+\tau}\right)-\Delta\left(s, x_{t}\right)-\tau D_{2, e} \Delta\left(s, x_{t}\right) \partial x_{t} \\
\quad=\tau \int_{0}^{1}\left(D_{2} \Delta\left(s, x_{t}+\theta\left(x_{t+\tau}-x_{t}\right)\right) \frac{1}{\tau}\left(x_{t+\tau}-x_{t}\right)-D_{2, e} \Delta\left(s, x_{t}\right) \partial x_{t}\right) d \theta \\
=\tau \int_{0}^{1}\left(D_{2, e} \Delta\left(s, x_{t}+\theta\left(x_{t+\tau}-x_{t}\right)\right) \frac{1}{\tau}\left(x_{t+\tau}-x_{t}\right)-D_{2, e} \Delta\left(s, x_{t}\right) \partial x_{t}\right) d \theta .
\end{gathered}
$$

For $0 \neq \tau \rightarrow 0$ we have

$$
\left\|\frac{1}{\tau}\left(x_{t+\tau}-x_{t}\right)-\partial x_{t}\right\|_{C} \rightarrow 0 .
$$

Using this and hypothesis $(\mathrm{H})$ we infer that for $0 \neq \tau \rightarrow 0$ the last integrand converges to $0 \in \mathbb{R}^{k}$, uniformly with respect to $\theta \in[0,1]$. This yields the assertion. 4. From Eq. (3.1) we also infer that the map $D_{2} \Delta_{x}$ is continuous. It follows that $\Delta_{x}$ is continuously differentiable. For $0<t<t_{e}$ we have

$$
0=\Delta\left(r(t), x_{t}\right)=\Delta_{x}(r(t), t) \quad \text { and } \quad 0 \neq \operatorname{det} D_{1} \Delta_{x}(r(t), t) .
$$

Using the Implicit Function Theorem in a neighbourhood of $\left(r\left(t_{0}\right), t_{0}\right)$, for any $t_{0} \in\left(0, t_{e}\right)$, we find that $r \mid\left(0, t_{e}\right)$ is continuously differentiable, with

$$
\begin{aligned}
r^{\prime}(t) & =-\left(D_{1} \Delta_{x}(r(t), t)\right)^{-1}\left(D_{2} \Delta_{x}(r(t), t) 1\right) \\
& =-\left(D_{1} \Delta\left(r(t), x_{t}\right)\right)^{-1}\left(D_{2, e} \Delta\left(r(t), x_{t}\right) \partial x_{t}\right) \\
& =A\left(r(t), x_{t}, \partial x_{t}\right)
\end{aligned}
$$

for every $t \in\left(0, t_{e}\right)$. Finally, the preceding formula and a continuity argument yield that $r$ has a right derivative at $t=0$, and that $r^{\prime}$ is continuous also at $t=0$. 
In general the map

$$
A_{M}: M \ni(s, \phi) \mapsto A(s, \phi, \partial \phi) \in \mathbb{R}^{k}
$$

which appears on the right hand side of the differential equation in the Proposition 3.4 is not continuously differentiable. See, for example, the expression of the map $A$ in terms of the function $d$ in section 8 below. - In general lack of smoothness of $A_{M}$ precludes the possibility to study the modification of the initial value problem (1.2)-(1.4) where the algebraic equation (1.3) is replaced by $r^{\prime}(t)=A_{M}\left(r(t), x_{t}\right)$, in the framework of $[23,24,6]$.

Proposition 3.5. Any two solutions of the initial value problem (1.2)-(1.4) with $(s, \phi) \in M$ coincide on the intersection of their domains.

Proof. Consider two solutions $(r, x),(\hat{r}, \hat{x})$ with common domain $[0, T)$. Suppose $(r(t), x(t)) \neq(\hat{r}(t), \hat{x}(t))$ for some $t \in(0, T)$. Then

$$
t_{i}=\inf \{t \in(0, T):(r(t), x(t)) \neq(\hat{r}(t), \hat{x}(t))\}
$$

belongs to $[0, T)$, and $r\left(t_{i}\right)=\hat{r}\left(t_{i}\right), x_{t_{i}}=\hat{x}_{t_{i}}$. Let $s_{0}=r\left(t_{i}\right), \phi_{0}=x_{t_{i}}$. Then $\left(s_{0}, \phi_{0}\right) \in M \subset U$, and $\Delta\left(s_{0}, \phi_{0}\right)=0$, det $D_{1} \Delta\left(s_{0}, \phi_{0}\right) \neq 0$. There exist open neighbourhoods $N_{0} \subset C^{1}$ of $\phi_{0}$ and $V_{0} \subset(-h, 0)^{k}$ of $s_{0}$ with $V_{0} \times N_{0} \subset U$, and a continuously differentiable map $\sigma_{0}: N_{0} \rightarrow V_{0}$ so that (2.1)-(2.3) hold. We have $\phi_{0} \in X_{\sigma_{0}}$ since

$$
\phi_{0}^{\prime}(0)=g\left(s_{0}, \phi_{0}\right)=g\left(\sigma_{0}\left(\phi_{0}\right), \phi_{0}\right)=g_{\sigma_{0}}\left(\phi_{0}\right) .
$$

The initial value problem (2.4)-(2.5) with $\phi=\phi_{0} \in X_{\sigma_{0}}$ has a continuously differentiable solution $y:\left[-h, t_{y}\right) \rightarrow \mathbb{R}^{n}, 0<t_{y} \leq \infty$. By continuity there exists $\epsilon \in\left(0, t_{y}\right)$ so that for $0 \leq t<\epsilon$,

$$
\left(r\left(t_{i}+t\right), x_{t_{i}+t}\right) \in V_{0} \times N_{0} \ni\left(\hat{r}\left(t_{i}+t\right), \hat{x}_{t_{i}+t}\right) .
$$

Consider $\rho:[0, \epsilon) \ni t \mapsto r\left(t_{i}+t\right) \in(-h, 0)^{k}$ and $\xi:[-h, \epsilon) \ni t \mapsto x\left(t_{i}+t\right) \in \mathbb{R}^{n}$. We have $\left(\rho(t), \xi_{t}\right) \in V_{0} \times N_{0}$ and $\Delta\left(\rho(t), \xi_{t}\right)=0$ on $[0, \epsilon)$. Hence $\rho(t)=\sigma_{0}\left(\xi_{t}\right)$ on $[0, \epsilon)$. Also,

$$
\xi^{\prime}(t)=x^{\prime}\left(t_{i}+t\right)=g\left(r\left(t_{i}+t\right), x_{t_{i}+t}\right)=g\left(\rho(t), \xi_{t}\right)=g\left(\sigma_{0}\left(\xi_{t}\right), \xi_{t}\right)=g_{\sigma_{0}}\left(\xi_{t}\right)
$$

for $0<t<\epsilon$, and $\xi_{0}=x_{t_{i}}=\phi_{0}$. By uniqueness of solutions of the initial value problem (2.4)-(2.5), $y(t)=\xi(t)=x\left(t_{i}+t\right)$ for $-h \leq t<\epsilon$. Moreover, $r\left(t_{i}+t\right)=\rho(t)=\sigma_{0}\left(\xi_{t}\right)=\sigma_{0}\left(y_{t}\right)$ on $[0, \epsilon)$. For $\hat{x}$ and $\hat{r}$ we get the same result. So $r(t)=\hat{r}(t)$ on $\left[0, t_{i}+\epsilon\right)$ and $x(t)=\hat{x}(t)$ on $\left[-h, t_{i}+\epsilon\right)$, in contradiction to the properties of the infimum $t_{i}$.

For $(s, \phi) \in M$ set

$$
\begin{aligned}
t_{s, \phi}=t_{(s, \phi)}= & \sup \left\{t_{e} \in(0, \infty) \cup\{\infty\}:\left[0, t_{e}\right)\right. \text { is the domain of a } \\
& \text { solution of the initial value problem }(1.2)-(1.4)\} \\
& \leq \infty
\end{aligned}
$$

For every $(s, \phi) \in M$ there exists a maximal solution $\left(r^{s, \phi}, x^{s, \phi}\right)$ of the initial value problem (1.2)-(1.4) with domain $\left[0, t_{s, \phi}\right)$, which has the property that for any other solution $(r, x)$ of the same initial value problem, with domain $\left[0, t_{e}\right)$, we have $t_{e} \leq t_{s, \phi}$, and $r$ and $x$ are restrictions of $r^{s, \phi}$ and $x^{s, \phi}$, respectively. Let

$$
\Omega=\left\{(t, s, \phi) \in[0, \infty) \times M: t<t_{s, \phi}\right\}
$$


and define $F: \Omega \rightarrow M$ by

$$
F(t, s, \phi)=\left(r^{s, \phi}(t), x_{t}^{s, \phi}\right) .
$$

For $t \geq 0$ set

$$
\Omega_{t}=\{(s, \phi) \in M:(t, s, \phi) \in \Omega\}=\left\{(s, \phi) \in M: t<t_{s, \phi}\right\} .
$$

In case $\Omega_{t} \neq \emptyset$ the map $F_{t}: \Omega_{t} \rightarrow M$ is given by

$$
F_{t}(s, \phi)=F(t, s, \phi) .
$$

Obviously, $\Omega_{0}=M$ and $F_{0}=\operatorname{id}_{M}$.

Notice that for every $(s, \phi) \in M$ the flowline $\left[0, t_{s, \phi}\right) \ni t \mapsto F(t, s, \phi) \in M$ is continuous (with the continuity of the map $\left[0, t_{s, \phi}\right) \ni t \mapsto x_{t}^{s, \phi} \in C^{1}$ ).

Proposition 3.6. (i) For $0 \leq t<t_{s, \phi}$ and $0 \leq u<t_{F(t, s, \phi)}, t+u<t_{s, \phi}$ and

$$
F(t+u, s, \phi)=F(u, F(t, s, \phi)) \text {. }
$$

(ii) For every $t \geq 0$ the set $\Omega_{t}$ is an open subset of $M . \Omega$ is an open subset of $[0, \infty) \times M$ (with the topology given by the norm on $\mathbb{R} \times B$ ), and $F$ is continuous.

Proof. 1. Proof of (i). Let $(r, x)=\left(r^{s, \phi}, x^{s, \phi}\right), t_{1}=t_{s, \phi},(u, \psi)=F(t, s, \phi),(v, y)=$ $\left(r^{u, \psi}, x^{u, \psi}\right), t_{2}=t_{u, \psi}$. So, $v(0)=u=r(t)$ and $y(a)=x(t+a)$ on [-h,0]. Define $w:\left[0, t+t_{2}\right) \rightarrow \mathbb{R}^{k}$ and $z:\left[-h, t+t_{2}\right) \rightarrow \mathbb{R}^{n}$ by $w(a)=r(a)$ on $[0, t]$ and $w(a)=v(a-t)$ on $\left[t, t+t_{2}\right), z(a)=x(a)$ on $[-h, t], z(a)=y(a-t)$ on $\left[t, t+t_{2}\right)$. Then $w$ is continuous and $z$ is continuously differentiable. On $\left(t, t+t_{2}\right)$ we have $z_{a}=y_{a-t}$. It follows that

$$
\begin{aligned}
& \Delta\left(w(a), z_{a}\right)=\Delta\left(r(a), x_{a}\right)=0 \quad \text { on } \quad[0, t], \\
& \Delta\left(w(a), z_{a}\right)=\Delta\left(v(a-t), y_{a-t}\right)=0 \quad \text { on }\left[t, t+t_{2}\right),
\end{aligned}
$$

and

$$
\begin{aligned}
z^{\prime}(a)=x^{\prime}(a) & =g\left(r(a), x_{a}\right)=g\left(w(a), z_{a}\right) \quad \text { on } \quad(0, t], \\
z^{\prime}(a)=y^{\prime}(a-t) & =g\left(v(a-t), y_{a-t}\right)=g\left(w(a), z_{a}\right) \quad \text { on } \quad\left(\left[t, t+t_{2}\right) .\right.
\end{aligned}
$$

Therefore, $t+t_{2}<t_{s, \phi}, t+u \in\left[0, t_{s, \phi}\right)$, and

$$
\begin{aligned}
F(t+u, s, \phi) & =\left(w(t+u), z_{t+u}\right) \\
& =\left(v(t+u-t), y_{t+u-t}\right)=\left(v(u), y_{u}\right) \\
& =F\left(u, v(0), y_{0}\right)=F\left(u, r(t), x_{t}\right)=F(u, F(t, s, \phi)) .
\end{aligned}
$$

2. Proof of (ii).

2.1. Proof that for every $\left(s_{0}, \phi_{0}\right) \in M$ there exist $t_{0}>0$ and an open neighbourhood $W$ of $\left(s_{0}, \phi_{0}\right)$ in $M$ so that $\left[0, t_{0}\right] \times W \subset \Omega$ and $F \mid\left(\left[0, t_{0}\right] \times W\right)$ is continuous: Let $\left(s_{0}, \phi_{0}\right) \in M$ be given. Then (2.7) holds, and there exist $V_{0}, N_{0}, \sigma_{0}, X_{\sigma_{0}}, F_{\sigma_{0}}$ and $N_{01}, t_{0}>0$ as in Propositions 2.1 and 3.3. It follows that

$$
\begin{aligned}
{\left[0, t_{0}\right] \times\left(\left(V_{0} \times N_{01}\right) \cap M\right) \subset } & \Omega, \\
F(t, s, \phi)= & \left(\sigma_{0}\left(F_{\sigma_{0}}(t, \phi)\right), F_{\sigma_{0}}(t, \phi)\right) \\
& \text { on }\left[0, t_{0}\right] \times\left(\left(V_{0} \times N_{01}\right) \cap M\right),
\end{aligned}
$$

and the restriction of $F$ to $\left[0, t_{0}\right] \times\left(\left(V_{0} \times N_{01}\right) \cap M\right)$ is continuous.

2.2. We show that $\Omega_{t} \subset M$ is open and that $F_{t}$ is continuous, for $t \geq 0$ and $\Omega_{t} \neq \emptyset$. 
In case $t=0$ we have $\Omega_{t}=M$ and $F_{t}=\mathrm{id}_{M}$. Consider the case $t>0$. Let $\left(r_{0}, \phi_{0}\right) \in \Omega_{t}$ be given. Then $t<t_{r_{0}, \phi_{0}}$. Due to the continuity of flowlines the set $K=F\left([0, t] \times\left\{\left(r_{0}, \phi_{0}\right)\right\}\right) \subset M$ is compact. Using this and part 2.1 we find $u>0$ and an open neighbourhood $N$ of $K$ in $M$ so that $[0, u] \times N \subset \Omega$ and $F$ is continuous on $[0, u] \times N$. Choose $J \in \mathbb{N}$ with $\frac{t}{J}<u$. Then $N \subset \Omega_{t / J}$, and $F_{t / J}$ is continuous on $N$. Define $\left(r_{j}, \phi_{j}\right) \in K$ for $j=1, \ldots, J$ by

$$
\left(r_{j}, \phi_{j}\right)=F_{t / J}\left(r_{j-1}, \phi_{j-1}\right) \text {. }
$$

By assertion (i) and induction, $\left(r_{J}, \phi_{J}\right)=F_{t}\left(r_{0}, \phi_{0}\right)$. Let an open neighbourhood $N_{J} \subset M$ of $\left(r_{J}, \phi_{J}\right)$ be given. Recursively we find open neighbourhoods $N_{j} \subset N \subset$ $M$ of $\left(r_{j}, \phi_{j}\right), j=J-1, \ldots, 0$ so that $F_{t / J}\left(N_{j}\right) \subset N_{j+1}$ for these $j$. Using (i) and induction once again we infer $N_{0} \subset \Omega_{t}$ and $F_{t}\left(N_{0}\right) \subset N_{J}$.

2.3. Proof that $\Omega \subset[0, \infty) \times M$ is open. Let $(t, s, \phi) \in \Omega$ be given. Choose $u \in\left(t, t_{s, \phi}\right)$. Then $(s, \phi) \in \Omega_{u}$. As $\Omega_{u} \subset M$ is open there is a neighbourhood $N \subset M$ of $(s, \phi)$ with $N \subset \Omega_{u}$. Then $[0, u] \times N$ is a neighbourhood of $(t, s, \phi)$ in $[0, \infty) \times M$ which is contained in $\Omega$.

2.4. Proof that $F$ is continuous. Let $\left(t_{0}, s_{0}, \phi_{0}\right) \in \Omega$ be given. Part 2.1 yields continuity at $\left(t_{0}, s_{0}, \phi_{0}\right)$ in case $t_{0}=0$. Assume $t_{0}>0$. By part 2.1 there exists $u \in\left(0, t_{0}\right)$ so that $F$ is continuous at $\left(u, s_{0}, \phi_{0}\right)$. Let a neighbourhood $N_{0} \subset M$ of $F\left(t_{0}, s_{0}, \phi_{0}\right)$ be given. As $\Omega_{t_{0}-u} \subset M$ is open and $F_{t_{0}-u}$ is continuous there exists a neighbourhood $N_{1} \subset M$ of $F\left(u, s_{0}, \phi_{0}\right)$ with $F_{t_{0}-u}\left(N_{1}\right) \subset N_{0}$. Moreover, $F$ maps a neighbourhood $(u-\delta, u+\delta) \times N_{2}, \delta \in(0, u)$ and $N_{2} \subset M$ open with $\left(s_{0}, \phi_{0}\right) \in N_{2}$, of $\left(u, s_{0}, \phi_{0}\right)$ in $\Omega$ into $N_{1}$. For each $(t, s, \phi)$ in the neighbourhood $\left(t_{0}-\delta, t_{0}+\delta\right) \times N_{2}$ of $\left(t_{0}, s_{0}, \phi_{0}\right)$ in $[0, \infty) \times M$ we have $t=\left(t_{0}-u\right)+\left(u+t-t_{0}\right)$. Using (i) we infer $(t, s, \phi) \in \Omega$ and

$$
F(t, s, \phi)=F\left(t_{0}-u, F\left(u+t-t_{0}, s, \phi\right)\right) \subset F_{t_{0}-u}\left(N_{1}\right) \subset N_{0} .
$$

It may be convenient for later use to state the relation between the semiflow $F$ for the algebraic-delay differential system and the semiflows for delay differential equations given by Proposition 2.1 in terms of flow equivalence. So let $\left(s_{0}, \phi_{0}\right) \in M$ be given and consider $V_{0}, N_{0}, \sigma_{0}, X_{\sigma_{0}}, F_{\sigma_{0}}$ as in Proposition 2.1. The set

$$
M_{0}=M \cap\left(V_{0} \times N_{0}\right)
$$

is open in $M$. For the projection $p_{2}: B \rightarrow C^{1}$ we easily obtain

$$
p_{2} M_{0}=X_{\sigma_{0}}
$$

and the continuously differentiable map

$$
P: M_{0} \stackrel{p_{2}}{\rightarrow} X_{\sigma_{0}}
$$

is a diffeomorphism whose inverse is the restricted graph map

$$
G: X_{\sigma_{0}} \ni \phi \mapsto\left(\sigma_{0}(\phi), \phi\right) \in M_{0} .
$$

We define the restricted semiflow $F_{0}: \Omega_{0} \rightarrow M_{0}$ on $M_{0}$ by the relations

$$
\begin{aligned}
t_{0, s, \phi} & =\sup \left\{t \in\left(0, t_{s, \phi}\right): F(u, s, \phi) \in V_{0} \times N_{0} \text { for } 0 \leq u \leq t\right\} \\
& \leq t_{s, \phi} \leq \infty, \text { for }(s, \phi) \in M_{0}, \\
\Omega_{0} & =\left\{(t, s, \phi) \in[0, \infty) \times M_{0}: t<t_{0, s, \phi}\right\}, \\
F_{0}(t, s, \phi) & =F(t, s, \phi) .
\end{aligned}
$$


Recall the notation $t_{\sigma_{0}, \phi}$ for the upper bound of the domain of the maximal solution $x^{\sigma_{0}, \phi}, \phi \in X_{\sigma_{0}}$, from Proposition 2.1. A comparison of maximal solutions of the relevant initial value problems yields the following result on continuously differentiable (semi-) flow equivalence.

Proposition 3.7. For each $(s, \phi) \in M_{0}$,

$$
t_{0, s, \phi}=t_{\sigma_{0}, P(s, \phi)},
$$

and for $0 \leq t<t_{0, s, \phi}$,

$$
P F_{0}(t, s, \phi)=F_{\sigma_{0}}(t, P(s, \phi)) \text {. }
$$




\section{Smoothness of SOlution operators}

We begin the proof that all maps $F_{t}, t \geq 0$ and $\Omega_{t} \neq \emptyset$, are continuously differentiable with results about the variational equation

$$
v^{\prime}(t)=L\left(F(t, s, \phi), v_{t}\right)
$$

along flowlines where the continuous map

$$
L: M \times C \rightarrow \mathbb{R}^{n}
$$

is defined by the equation

$L(s, \phi, \chi)=\Lambda(s, \phi, A(s, \phi, \chi), \chi) \quad\left(=D_{e} g(s, \phi)\left(-\left(D_{1} \Delta(s, \phi)\right)^{-1}\left(D_{2, e} \Delta(s, \phi) \chi\right), \chi\right)\right)$.

For each $(s, \phi) \in M$ the linear map

$$
L_{1}(s, \phi): C^{1} \ni \chi \mapsto L(s, \phi, \chi) \in \mathbb{R}^{n}
$$

satisfies

$$
L_{1}(s, \phi) \chi=D g(s, \phi)\left(A_{1}(s, \phi) \chi, \chi\right)
$$

for all $\chi \in C^{1}$, and is continuous.

Proposition 4.1. (i) Every compact set $K \subset M$ has a neighbourhood $N$ in $M$ with

$$
\sup _{(s, \phi) \in N}\|L(s, \phi, \cdot)\|_{L_{c}\left(C, \mathbb{R}^{n}\right)}<\infty .
$$

(ii) The map $L_{1}: M \rightarrow L_{c}\left(C^{1}, \mathbb{R}^{n}\right)$ is continuous.

Proof. 1. Proof of (i): Each $(s, \phi, 0) \in K \times\{0\} \subset M \times C$ has a neighbourhood in $M \times C$ on which the continuous map $L$ is bounded. Due to compactness of $K \times\{0\} \subset M \times C$ a finite collection of these neighbourhoods covers $K \times\{0\}$. This yields a neighbourhood $N$ of $K$ in $M$ and a neighbourhood $V$ of 0 in $C$ so that $L$ is bounded on $N \times V$, and the assertion follows.

2. Proof of (ii): For $(s, \phi)$ and $\left(s_{0}, \phi_{0}\right)$ in $M$ and for every $\chi \in C^{1}$ with $\|\chi\|_{C^{1}} \leq 1$, set $p=A_{1}(s, \phi) \chi$ and $\left.p_{0}=A_{1}\left(s_{0}, \phi_{0}\right) \chi\right)$. Then we have

$$
\begin{gathered}
\left|L_{1}(s, \phi) \chi-L_{1}\left(s_{0}, \phi_{0}\right) \chi\right|=\left|D g(s, \phi)(p, \chi)-D g\left(s_{0}, \phi_{0}\right)\left(p_{0}, \chi\right)\right| \\
\leq\left|D g(s, \phi)(p, \chi)-D g\left(s_{0}, \phi_{0}\right)(p, \chi)\right|+\left|D g\left(s_{0}, \phi_{0}\right)(p, \chi)-D g\left(s_{0}, \phi_{0}\right)\left(p_{0}, \chi\right)\right| \\
\leq\left\|D g(s, \phi)-D g\left(s_{0}, \phi_{0}\right)\right\|_{L_{c}(B, \mathbb{R})}(|p|+1)+\left|D g\left(s_{0}, \phi_{0}\right)\left(p-p_{0}, 0\right)\right| \\
\leq\left\|D g(s, \phi)-D g\left(s_{0}, \phi_{0}\right)\right\|_{L_{c}\left(B, \mathbb{R}^{n}\right)}\left(\left|A_{1}(s, \phi) \chi\right|+1\right) \\
\quad+\left\|D g\left(s_{0}, \phi_{0}\right)\right\|_{L_{c}\left(B, \mathbb{R}^{n}\right)}\left|A_{1}(s, \phi) \chi-A_{1}\left(s_{0}, \phi_{0}\right) \chi\right| \\
\leq\left\|D g(s, \phi)-D g\left(s_{0}, \phi_{0}\right)\right\|_{L_{c}\left(B, \mathbb{R}^{n}\right)}\left(\left\|A_{1}(s, \phi)\right\|_{L_{c}\left(C^{1}, \mathbb{R}^{k}\right)}+1\right) \\
+\left\|D g\left(s_{0}, \phi_{0}\right)\right\|_{L_{c}\left(B, \mathbb{R}^{n}\right)} \| A_{1}(s, \phi)-A_{1}\left(s_{0}, \phi_{0} \|_{L_{c}\left(C^{1}, \mathbb{R}^{k}\right)} .\right.
\end{gathered}
$$

This estimate and the continuity of $A_{1}$ combined yield the assertion.

Proposition 4.2. Let $(s, \phi) \in M, r=r^{s, \phi}, x=x^{s, \phi}$, and $\chi \in C$. There exists a unique continuous function $v:\left[-h, t_{s, \phi}\right) \rightarrow \mathbb{R}^{n}$ which is differentiable for $0<t<$ $t_{s, \phi}$ and satisfies

$$
\begin{aligned}
v^{\prime}(t)= & L\left(F(t, s, \phi), v_{t}\right) \\
& \text { for } 0<t<t_{s, \phi} \quad \text { and } \\
v_{0}= & \chi .
\end{aligned}
$$

In case $(p, \chi) \in T_{(s, \phi)} M$ the function $v=v^{s, \phi, \chi}$ is continuously differentiable. 
Proof. Using Proposition 4.1 (i) and the continuity of flowlines we can proceed as in the proofs of Propositions 1-3 and Corollary 1 in section 2 of [24] and obtain that for every $\chi \in C$ there is a unique continuous function $v:\left[-h, t_{s, \phi}\right) \rightarrow \mathbb{R}^{n}$ with $v_{0}=\chi$ which is differentiable for $0<t<t_{s, \phi}$ and satisfies $v^{\prime}(t)=L\left(F(t, s, \phi), v_{t}\right)$ for such $t$. Continuity of flowlines and continuity of the curve $\left[0, t_{s, \phi}\right) \ni t \mapsto v_{t} \in C$ combined yield

$$
\lim _{t \searrow 0} v^{\prime}(t)=\lim _{t \searrow 0} L\left(F(t, s, \phi), v_{t}\right)=L(s, \phi, \chi) .
$$

In case $(p, \chi) \in T_{(s, \phi)} M$ we have $\chi \in C^{1}$ and $\chi^{\prime}(0)=D g(s, \phi)\left(A_{1}(s, \phi) \chi, \chi\right)=$ $L(s, \phi, \chi)$, by Corollary 3.2. We obtain $\lim _{t \nearrow_{0}} v^{\prime}(t)=\lim _{t \searrow_{0}} v^{\prime}(t)$, which implies that $v$ is differentiable at 0 , and that $v^{\prime}$ is continuous.

Proposition 4.3. Each map $F_{t}, 0 \leq t<\infty$ and $\Omega_{t} \neq \emptyset$, is continuously differentiable. For every $(t, s, \phi) \in \Omega$ and $(p, \chi) \in T_{(s, \phi)} M$ we have

$$
D F_{t}(s, \phi)(p, \chi)=\left(q^{s, \phi, \chi}(t), v_{t}^{s, \phi, \chi}\right)
$$

where $q^{s, \phi, \chi}:\left[0, t_{s, \phi}\right) \rightarrow \mathbb{R}^{k}$ is given by

$$
q^{s, \phi, \chi}(u)=A_{1}\left(r^{s, \phi}(u), x_{u}^{s, \phi}\right) v_{u}^{s, \phi, \chi} .
$$

Proof. 1. We begin with a local result and show that for every $\left(s_{0}, \phi_{0}\right) \in M$ there exist $t_{0}>0$ and an open neighbourhood $N$ of $\left(s_{0}, \phi_{0}\right)$ in $M$ so that $\left[0, t_{0}\right] \times N \subset \Omega$, for every $t \in\left[0, t_{0}\right]$ the map $F_{t} \mid N$ is continuously differentiable, and (4.3) and (4.4) hold for all $(s, \phi) \in N,(p, \chi) \in T_{(s, \phi)} M$. Proof: Let $\left(s_{0}, \phi_{0}\right) \in M$ be given. Then we have (2.7), and there are $V_{0}, N_{0}, \sigma_{0}, X_{\sigma_{0}}, F_{\sigma_{0}}$ and $N_{01}, t_{0}>0$ as in Propositions 2.1 and 3.3. As in part 2.1 of the proof of Proposition 3.6 we get (3.2) and (3.3). Using Proposition 2.1 we infer that for $t \in\left[0, t_{0}\right]$ the restriction of $F_{t}$ to $\left(V_{0} \times N_{01}\right) \cap M$ is continuously differentiable, and that for $(s, \phi) \in\left(V_{0} \times N_{01}\right) \cap M,(p, \chi) \in T_{(s, \phi)} M$, and $r=r^{s, \phi}, x=x^{s, \phi}$ we have

$$
\begin{aligned}
D F_{t}(s, \phi)(p, \chi) & =\left(D\left(\sigma_{0} \circ F_{\sigma_{0}, t}\right)(\phi) \chi, D F_{\sigma_{0}, t}(\phi) \chi\right) \\
& =\left(D \sigma_{0}\left(x_{t}\right) v_{t}, v_{t}\right) \\
& =\left(-\left(D_{1} \Delta\left(\sigma_{0}\left(x_{t}\right), x_{t}\right)\right)^{-1}\left(D_{2} \Delta\left(\sigma_{0}\left(x_{t}\right), x_{t}\right) v_{t}\right), v_{t}\right) \\
& =\left(A_{1}\left(\sigma_{0}\left(x_{t}\right), x_{t}\right) v_{t}, v_{t}\right) \\
& =\left(A_{1}\left(r(t), x_{t}\right) v_{t}, v_{t}\right)
\end{aligned}
$$

with a continuously differentiable solution $v:\left[-h, t_{\sigma_{0}, \phi}\right) \rightarrow \mathbb{R}^{n}$ of the IVP (2.8)(2.9). The inequality $t_{\sigma_{0}, \phi} \leq t_{s, \phi}$ holds, and for $0<u<t_{\sigma_{0}, \phi}$ we get

$$
\begin{aligned}
v^{\prime}(u) & =D g_{\sigma_{0}}\left(x_{u}\right) v_{u} \\
& =D g\left(\sigma_{0}\left(x_{u}\right), x_{u}\right)\left(D \sigma_{0}\left(x_{u}\right) v_{u}, v_{u}\right) \\
& =D g\left(\sigma_{0}\left(x_{u}\right), x_{u}\right)\left(-\left(D_{1} \Delta\left(\sigma_{0}\left(x_{u}\right), x_{u}\right)\right)^{-1}\left(D_{2} \Delta\left(\sigma_{0}\left(x_{u}\right), x_{u}\right) v_{u}\right), v_{u}\right) \\
& =L\left(\sigma_{0}\left(x_{u}\right), x_{u}, v_{u}\right) \\
& =L\left(r(u), x_{u}, v_{u}\right)=L\left(F(u, s, \phi), v_{u}\right),
\end{aligned}
$$

which is (4.1). Proposition 4.2 guarantees $v(u)=v^{s, \phi, \chi}(u)$ on $\left[-h, t_{\sigma_{0}, \phi}\right)$, and we infer that (4.3) and (4.4) hold for $t \in\left[0, t_{0}\right]$.

2. For $t=0, F_{t}=\operatorname{id}_{M}$. Let $t>0$ and $\left(s_{0}, \phi_{0}\right) \in \Omega_{t}$ be given. Set $(r, x)=$ $\left(r^{s_{0}, \phi_{0}}, x^{s_{0}, \phi_{0}}\right)$. Consider the compact set $K=\left\{F\left(u, s_{0}, \phi_{0}\right): 0 \leq u \leq t\right\} \subset M$. Part 1 says that for every $u \in[0, t]$ there exist $t_{u}>0$ and an open neighbourhood $N_{u}$ of $F\left(u, s_{0}, \phi_{0}\right)$ in $M$ with $\left[0, t_{u}\right] \times N_{u} \subset \Omega$ such that for every $w \in\left[0, t_{u}\right]$ the 
restriction $F_{w} \mid N_{u}$ is continuously differentiable. Recall Corollary 3.2, for $(s, \phi) \in$ $N_{u}$. The compact set $K$ is covered by a finite collection of the neighbourhoods $N_{u}$, say, by the union of $N_{u_{\nu}}, \nu=1, \ldots, \nu_{*}$, with $u_{\nu} \in[0, t]$. Choose $J \in \mathbb{N}$ with $t / J \leq \min _{\nu=1, \ldots, \nu_{*}} t_{u_{\nu}}$ and set $\left(s_{j}, \phi_{j}\right)=F\left(\frac{j t}{J}, s_{0}, \phi_{0}\right)$ for $j=1, \ldots, J$. For every $j \in\{0, \ldots, J-1\}$ there exists $\nu_{j} \in\left\{1, \ldots, \nu_{*}\right\}$ with $\left(s_{j}, \phi_{j}\right) \in N_{u_{\nu_{j}}}$. Set $N_{j}=N_{u_{\nu_{j}}} . N_{j}$ is an open neighbourhood of $\left(s_{j}, \phi_{j}\right)$ in $M$ with $N_{j} \subset \Omega_{t / J}$, $F_{t / J} \mid N_{j}$ is continuously differentiable, and we have

$$
D F_{t / J}\left(s_{j}, \phi_{j}\right)(p, \chi)=\left(q^{s_{j}, \phi_{j}, \chi}(t / J), v_{t / J}^{s_{j}, \phi_{j}, \chi}\right)
$$

for all $(p, \chi) \in T_{\left(s_{j}, \phi_{j}\right)} M$, with $q^{s_{j}, \phi_{j}, \chi}$ defined by Eq. (4.4), with $\left(s_{j}, \phi_{j}\right)$ in place of $(s, \phi)$.

3. We proceed as in part 2.2 of the proof of Proposition 3.6 and obtain open neighbourhoods $\hat{N}_{j} \subset \Omega_{t / J}$ of $\left(s_{j}, \phi_{j}\right)$ for $j=0, \ldots, J-1$ so that $F_{t / J} \mid \hat{N}_{j}$ is continuously differentiable and $F_{t / J}\left(\hat{N}_{j}\right) \subset \hat{N}_{j+1}$ for these $j$. It follows that $F_{t} \mid \hat{N}_{0}=$ $\left(F_{t / J}\right)^{J} \mid \hat{N}_{0}$ is continuously differentiable.

4. Let $(p, \chi) \in T_{\left(s_{0}, \phi_{0}\right)} M$ and set $v=v^{s_{0}, \phi_{0}, \chi}$. Define $q=q^{s_{0}, \phi_{0}, \chi}$ by Eq. (4.4) (with $\left(s_{0}, \phi_{0}\right)$ in place of $\left.(s, \phi)\right)$. For $j=1, \ldots, J$ set $\left(p_{j}, \chi_{j}\right)=\left(q(j t / J), v_{j t / J}\right)$. In order to prove Eq. (4.3) we show by induction that for $j=1, \ldots, J$ we have

$$
D F_{j t / J}\left(s_{0}, \phi_{0}\right)(p, \chi)=\left(p_{j}, \chi_{j}\right) .
$$

For $j=1$, this is Eq. (4.5). Suppose Eq. (4.6) holds for some $j \in\{1, \ldots, J-1\}$. Then $\left(p_{j}, \chi_{j}\right) \in T_{\left(s_{j}, \phi_{j}\right)} M$. By Proposition 3.6 (i),

$$
r^{s_{j}, \phi_{j}}(u)=r\left(u+\frac{j t}{J}\right) \quad \text { for } \quad 0 \leq u \leq \frac{t}{J}
$$

and

$$
x^{s_{j}, \phi_{j}}(u)=x\left(u+\frac{j t}{J}\right) \quad \text { for } \quad-h \leq u \leq \frac{t}{J} .
$$

The continuously differentiable function

$$
w:\left[-h, \frac{t}{J}\right] \ni u \mapsto v\left(u+\frac{j t}{J}\right) \in \mathbb{R}
$$

satisfies $w_{0}=v_{j t / J}=\chi_{j}$, and for $0<u \leq \frac{t}{J}$,

$$
\begin{aligned}
w^{\prime}(u) & =v^{\prime}\left(u+\frac{j t}{J}\right) \\
& =L\left(r\left(u+\frac{j t}{J}\right), x_{u+\frac{j t}{J}}, v_{u+\frac{j t}{J}}\right) \\
& =L\left(r^{s_{j}, \phi_{j}}(u), x_{u}^{s_{j}, \phi_{j}}, w_{u}\right) .
\end{aligned}
$$

By uniqueness (Proposition 4.2), w(u) $=v^{s_{j}, \phi_{j}, \chi_{j}}(u)$ on $\left[-h, \frac{t}{J}\right]$. Consequently, $v\left(u+\frac{j t}{J}\right)=v^{s_{j}, \phi_{j}, \chi_{j}}(u)$ on $\left[-h, \frac{t}{J}\right]$. In particular, $v_{(j+1) t / J}=v_{t / J}^{s_{j}, \phi_{j}, \chi_{j}}$. Using this and the definitions of $q$ and $q^{s_{j}, \phi_{j}, \chi_{j}}$ we also get

$$
q((j+1) t / J)=q^{s_{j}, \phi_{j}, \chi_{j}}(t / J)
$$


It follows that

$$
\begin{aligned}
D F_{(j+1) t / J}\left(s_{0}, \phi_{0}\right)(p, \chi) & =D F_{t / J}\left(s_{j}, \phi_{j}\right)\left(D F_{j t / J}\left(s_{0}, \phi_{0}\right)(p, \chi) \quad\right. \text { (chain rule) } \\
& =D F_{t / J}\left(s_{j}, \phi_{j}\right)\left(p_{j}, \chi_{j}\right) \quad \text { (by assumption) } \\
& =\left(q^{s_{j}, \phi_{j}, \chi_{j}}(t / J), v_{t / J}^{s_{j}, \phi_{j}, \chi_{j}}\right) \quad(\text { by }(4.5)) \\
& =\left(q((j+1) t / J), v_{(j+1) t / J}\right)=\left(p_{j+1}, \chi_{j+1}\right) .
\end{aligned}
$$




\section{The time Derivative}

In this section we show that flowlines have derivatives for $t>h$, and that the corresponding partial derivative of the semiflow is continuous. We consider the modified map

$$
\hat{F}: \Omega \ni(u, y, \psi) \mapsto F(u, y, \psi) \in B
$$

and flowlines

$$
\hat{F}_{y, \psi}:\left[0, t_{y, \psi}\right) \ni u \mapsto \hat{F}(u, y, \psi) \in B .
$$

Proposition 5.1. (i) Let $(y, \psi) \in M$ and $r=r^{y, \psi}, x=x^{y, \psi}, t_{e}=t_{y, \psi}$. The restriction $x^{\prime} \mid\left(0, t_{e}\right)$ is continuously differentiable, with

$$
x^{\prime \prime}(u)=L\left(F(u, y, \psi), \partial x_{u}\right)
$$

for $0<u<t_{e}$.

(ii) For $(y, \psi) \in M$ with $h<t_{y, \psi}$ the curve $\hat{F}_{y, \psi}$ is differentiable in $\left(h, t_{y, \psi}\right)$, with

$$
D \hat{F}_{y, \psi}(u) 1=\left(r^{\prime}(u), \partial x_{u}\right) \in B
$$

for $h<u<t_{y, \psi}, r=r^{y, \psi}, x=x^{y, \psi}$.

(iii) The map

$$
\left\{(u, y, \psi) \in \Omega: h<u<t_{y, \psi}\right\} \ni(u, y, \psi) \mapsto D \hat{F}_{y, \psi}(u) \in L_{c}(\mathbb{R}, B)
$$

is continuous.

Proof. 1. Proof of (i).

1.1. Proof that $x^{\prime}$ is differentiable at $u \in\left(0, t_{e}\right)$ and that Eq. (5.1) holds: For all real $w \neq 0$ with $u+w \in\left[-h, t_{e}\right)$ we have

$$
\begin{gathered}
\frac{1}{w}\left(x^{\prime}(u+w)-x^{\prime}(u)\right)-L\left(F(u, y, \psi), \partial x_{u}\right) \\
=\frac{1}{w}(g(F(u+w, y, \psi))-g(F(u, y, \psi)))-\Lambda\left(F(u, y, \psi), A\left(F(u, y, \psi), \partial x_{u}\right), \partial x_{u}\right) \\
=\frac{1}{w} \int_{0}^{1} D g(F(u, y, \psi)+\theta(F(u+w, y, \psi)-F(u, y, \psi))) \\
{\left[F(u+w, y, \psi)-(F(u, y, \psi)] d \theta-\Lambda\left(F(u, y, \psi), A\left(F(u, y, \psi), \partial x_{u}\right), \partial x_{u}\right)\right.} \\
=\int_{0}^{1}\left[D g(F(u, y, \psi)+\theta(F(u+w, y, \psi)-F(u, y, \psi))) \frac{1}{w}[F(u+w, y, \psi)-(F(u, y, \psi)]\right. \\
\left.-\Lambda\left(F(u, y, \psi), A\left(F(u, y, \psi), \partial x_{u}\right), \partial x_{u}\right)\right] d \theta . \\
=\int_{0}^{1}\left[D_{e} g(F(u, y, \psi)+\theta(F(u+w, y, \psi)-F(u, y, \psi))) \frac{1}{w}[F(u+w, y, \psi)-(F(u, y, \psi)]\right. \\
\left.-\Lambda\left(F(u, y, \psi), A\left(F(u, y, \psi), \partial x_{u}\right), \partial x_{u}\right)\right] d \theta \\
=\int_{0}^{1}\left[\Lambda(F(u, y, \psi)+\theta(F(u+w, y, \psi)-F(u, y, \psi))), \frac{1}{w}[F(u+w, y, \psi)-(F(u, y, \psi)])\right. \\
\left.-\Lambda\left(F(u, y, \psi), A\left(F(u, y, \psi), \partial x_{u}\right), \partial x_{u}\right)\right] d \theta .
\end{gathered}
$$

Let $\epsilon>0$. By the continuity of $\Lambda$ there exist a convex neighbourhood $U_{1}$ of $F(u, y, \psi)$ in $U$ and a neighbourhood $V$ of $\left(A\left(F(u, y, \psi), \partial x_{u}\right), \partial x_{u}\right)$ in $\mathbb{R}^{k} \times C$ so that for all $(\bar{s}, \bar{\phi}, p, \chi) \in U_{1} \times V$ we have

$$
\left.\mid \Lambda(\bar{s}, \bar{\phi}, p, \chi)-\Lambda\left(F(u, y, \psi), A\left(F(u, y, \psi), \partial x_{u}\right), \partial x_{u}\right)\right) \mid<\epsilon .
$$


By the continuity of flowlines there exist $u_{-}<u_{+}$with $0 \leq u_{-}<u<u_{+}<t_{e}$ so that for all $w \in \mathbb{R}$ with $u+w \in\left(u_{-}, u_{+}\right)$we have $F(u+w, y, \psi) \in U_{1}$. As $U_{1}$ is convex we get

$$
F(u, y, \psi)+\theta(F(u+w, y, \psi)-F(u, y, \psi)) \in U_{1}
$$

for such $w$ and for $0 \leq \theta \leq 1$. Using the uniform continuity of $r^{\prime}$ on $\left[0, u_{+}\right]$and the uniform continuity of $x^{\prime}$ on $\left[-h, u_{+}\right]$and the equations

$$
\begin{aligned}
\frac{1}{w}(r(u+w)-r(u))-r^{\prime}(u) & =\frac{1}{w} \int_{0}^{w}\left(r^{\prime}(u+\theta)-r^{\prime}(u)\right) d \theta, \\
\frac{1}{w}(x(u+w+a)-x(u+a))-x^{\prime}(u+a) & =\frac{1}{w} \int_{0}^{w}\left(x^{\prime}(u+\theta+a)-x^{\prime}(u+a)\right) d \theta
\end{aligned}
$$

for real $w \neq 0$ with $u+w \in\left[0, t_{e}\right)$ and for $a \in[-h, 0]$, and the equation for $r^{\prime}(u)$ in Proposition 3.4, we find an open interval $J \subset\left(u_{-}, u_{+}\right)$with $u \in J$ so that for all real $w \neq 0$ with $u+w \in J$ we have

$$
\frac{1}{w}[F(u+w, y, \psi)-F(u, y, \psi)]=\left(\frac{1}{w}(r(u+w)-r(u)), \frac{1}{w}\left(x_{u+w}-x_{u}\right)\right) \in V .
$$

For such $w$ we obtain

$$
\begin{gathered}
\left|\frac{1}{w}\left(x^{\prime}(u+w)-x^{\prime}(u)\right)-L\left(F(u, y, \psi), \partial x_{u}\right)\right| \\
\leq \int_{0}^{1} \mid \Lambda(F(u, y, \psi)+\theta(F(u+w, y, \psi)-F(u, y, \psi))), \frac{1}{w}[F(u+w, y, \psi)-(F(u, y, \psi)]) \\
-\Lambda\left(F(u, y, \psi), A\left(F(u, y, \psi), \partial x_{u}\right), \partial x_{u}\right) \mid d \theta<\epsilon,
\end{gathered}
$$

which yields the assertion.

1.2. The continuity of $x^{\prime \prime}:\left(0, t_{e}\right) \rightarrow \mathbb{R}^{n}$ follows from Eq. (5.1) since $L$, the flowline $\left[0, t_{e}\right) \ni u \mapsto F(u, y, \psi) \in M$, and the map $\left[0, t_{e}\right) \ni u \mapsto \partial x_{u} \in C$ are all continuous. 2. Proof of (ii). Let $(y, \psi) \in M$ with $h<t_{y, \psi}$ be given. Set $r=r^{y, \psi}, x=x^{y, \psi}, t_{e}=$ $t_{y, \psi}$. Due to Proposition 3.4 the component $p_{1} \circ F_{y, \psi}=r$ is differentiable. Consider the component $p_{2} \circ F_{y, \psi}$ and $h<u<t_{e}$. For $w \in \mathbb{R}$ with $u+w \in\left(h, t_{e}\right)$ we have

$$
\left\|p_{2} \circ F_{y, \psi}(u+w)-p_{2} \circ F_{y, \psi}(u)-w \partial x_{u}\right\|_{C^{1}}
$$

$=\left\|x_{u+w}-x_{u}-w \partial x_{u}\right\|_{C^{1}}=\left\|x_{u+w}-x_{u}-w \partial x_{u}\right\|_{C}+\left\|\partial x_{u+w}-\partial x_{u}-w \partial \partial x_{u}\right\|_{C}$, and for all $a \in[-h, 0]$,

$$
x_{u+w}(a)-x_{u}(a)-w\left(\partial x_{u}\right)(a)=\int_{0}^{w}\left(x^{\prime}(u+\theta+a)-x^{\prime}(u+a)\right) d \theta
$$

and

$$
\left(\partial x_{u+w}\right)(a)-\left(\partial x_{u}\right)(a)-w\left(\partial \partial x_{u}\right)(a)=\int_{0}^{w}\left(x^{\prime \prime}(u+\theta+a)-x^{\prime \prime}(u+a)\right) d \theta .
$$

Using the preceding equations and the uniform continuity of $x^{\prime}$ and of $x^{\prime \prime}$ on [ $u-$ $h-\delta, u+\delta] \subset\left(0, t_{e}\right)$, for $\delta>0$ sufficiently small, one verifies that $D\left(p_{2} \circ F_{y, \psi}\right)(u) \in$ $L_{c}\left(\mathbb{R}, C^{1}\right)$ exists and is given by $D\left(p_{2} \circ F_{y, \psi}\right)(u) 1=\partial x_{u} \in C^{1}$.

3. Proof of (iii).

3.1. Let $\Omega_{h<}=\left\{(t, s, \phi) \in \Omega: h<t<t_{s, \phi}\right\}$. It is enough to show that the maps

$$
\begin{gathered}
\rho: \Omega \ni(t, y, \psi) \mapsto\left(r^{y, \psi}\right)^{\prime}(t) \in \mathbb{R}^{k}, \\
\xi: \Omega \ni(t, y, \psi) \mapsto \partial x_{t}^{y, \psi} \in C,
\end{gathered}
$$


and

$$
\xi^{\prime}: \Omega_{h<} \ni(t, y, \psi) \mapsto \partial \partial x_{t}^{y, \psi} \in C
$$

are continuous. Continuity of $\xi$ is obvious from the continuity of $\hat{F}$. Proposition 3.4 gives $\rho(t, y, \psi)=A(F(t, y, \psi), \xi(t, y, \psi))$ on $\Omega$, which yields the continuity of $\rho$. 3.2. Proof that $\xi^{\prime}$ is continuous: By Eq. (5.1),

$$
\xi^{\prime}(u, y, \psi)(a)=\left(x^{y, \psi}\right)^{\prime \prime}(u+a)=L(F(u+a, y, \psi), \xi(u+a, y, \psi))
$$

for $(u, y, \psi) \in \Omega_{h<}$ and $-h \leq a \leq 0$. The continuity of $L, F$ and $\xi$ combined show that the map

$$
\hat{\xi}: \Omega \ni(w, y, \psi) \mapsto L(F(w, y, \psi), \xi(w, y, \psi)) \in \mathbb{R}^{n}
$$

is continuous. Let $\left(u_{0}, y_{0}, \psi_{0}\right) \in \Omega_{h<}$ be given, and let $\epsilon>0$. Choose $\delta_{0}>0$ with $h<u_{0}-\delta_{0}$ and $u_{0}+\delta_{0}<t_{y_{0}, \psi_{0}}$. Choose a neighbourhood $N$ of $\left(y_{0}, \psi_{0}\right)$ in the open subset $\Omega_{u_{0}+\delta_{0}}$ of $M$. Then $\left[0, u_{0}+\delta_{0}\right] \times N \subset \Omega$. The uniform continuity of $\hat{\xi}$ on the compact set $\left[u_{0}-\delta_{0}-h, u_{0}+\delta_{0}\right] \times\left\{\left(y_{0}, \psi_{0}\right)\right\}$ implies that there exists $\delta>0$ so that for all $u \in\left(u_{0}-\delta, u_{0}+\delta\right)$ and for all $(y, \psi) \in N$ with $\left\|(y, \psi)-\left(y_{0}, \psi_{0}\right)\right\|_{B}<\delta$, and for all $a \in[-h, 0]$, we have

$$
\epsilon>\left|\hat{\xi}(u+a, y, \psi)-\hat{\xi}\left(u_{0}+a, y_{0}, \psi_{0}\right)\right|=\left|\xi^{\prime}(u, y, \psi)(a)-\xi^{\prime}\left(u_{0}, y_{0}, \psi_{0}\right)(a)\right| .
$$

This yields the continuity of $\xi^{\prime}$ at $\left(u_{0}, y_{0}, \psi_{0}\right)$. 


\section{The VARIational EQUATION}

This section contains preparations for the proof that the semiflow $F$ is continuously differentiable on the subset of $\Omega$ given by $t>h$. Notice that because of this last restriction the proof can not be replaced by a simple reduction to a local version of the desired result (which would follow from section 2).

In section 7 we shall consider the modified map

$$
\hat{F}: \Omega \ni(t, s, \phi) \mapsto F(t, s, \phi) \in B
$$

and compositions $\hat{F} \circ K$ with local parametrizations (inverted manifold charts) $K: I \times V \rightarrow I \times N$ of the $C^{1}$-submanifold $\mathbb{R} \times M$ of the Banach space $\mathbb{R} \times B$, with $I \subset \mathbb{R}$ an open interval, $V$ an open subset of a tangent space $Y$ of $M$, and $N$ an open subset of $M$. Below we derive estimates which will be used in the proof that the partial derivatives $D_{Y}(\hat{F} \circ K)(t, p, \chi)$ with $t>h$ depend continuously on $(t, p, \chi) \in I \times V \subset \mathbb{R} \times Y \subset \mathbb{R} \times B$. We study solutions $v^{s, \phi, \chi}$ of the initial value problem (4.1)-(4.2). The proofs are adaptations of the proofs of Propositions 4-7 in [24].

Proposition 6.1. Let $(s, \phi) \in M$ and $0 \leq t<t_{s, \phi}$. There exists $c_{1}=c_{1}(t, s, \phi) \geq 0$ so that for each $\chi \in C$ the solution $v=v^{s, \phi, \chi}$ satisfies

$$
\left\|v_{u}\right\|_{C} \leq e^{c_{1} u}\|\chi\|_{C} \text { for all } u \in[0, t] .
$$

In case $h<t<t_{s, \phi}$ we have

$$
\mid v_{u}\left\|_{C^{1}} \leq c_{1} e^{c_{1} u}\right\| \chi \|_{C} \text { for all } u \in(h, t] .
$$

Proof. Proposition 4.1 (i) yields

$$
c=\sup _{0 \leq u \leq t}\|L(F(u, s, \phi), \cdot)\|_{L_{c}\left(C, \mathbb{R}^{n}\right)}<\infty .
$$

Let $v=v^{s, \phi, \chi}$. Using (4.1) and the continuity of the curve $[0, t] \ni w \mapsto v_{w} \in C$ we infer $|v(u)| \leq|v(0)|+c \int_{0}^{u}\left\|v_{w}\right\|_{C} d w$ for $0 \leq u \leq t$. Moreover, for such $u$ and for $-h \leq a \leq 0$,

$$
|v(u+a)| \leq\left\|v_{0}\right\|_{C}+c \int_{0}^{\max \{u+a, 0\}}\left\|v_{w}\right\|_{C} d w \leq\left\|v_{0}\right\|_{C}+c \int_{0}^{u}\left\|v_{w}\right\|_{C} d w
$$

Consequently,

$$
\left\|v_{u}\right\|_{C} \leq\left\|v_{0}\right\|_{C}+c \int_{0}^{u}\left\|v_{w}\right\|_{C} d w \text { on }[0, t]
$$

and by Gronwall's lemma,

$$
\left\|v_{u}\right\|_{C} \leq e^{c u}\left\|v_{0}\right\|_{C}=e^{c u}\|\chi\|_{C} \text { on }[0, t] .
$$

In case $h<t$ and $h<u \leq t$ we obtain

$$
\left\|\partial v_{u}\right\|_{C}=\max _{-h \leq a \leq 0}\left|v^{\prime}(u+a)\right|=\max _{-h \leq a \leq 0}\left|L\left(F(u+a, s, \phi), v_{u+a}\right)\right| \leq c e^{c u}\|\chi\|_{C} .
$$

Now the assertion becomes obvious.

Proposition 6.2. Let $(s, \phi) \in M$ and $0 \leq t<t_{s, \phi}$. There exist $c_{2}=c_{2}(t, s, \phi) \geq 0$ and a neighbourhood $N$ of $(s, \phi)$ in $M$ so that for every $(\bar{s}, \bar{\phi}) \in N$ we have $t<t_{\bar{s}, \bar{\phi}}$, and for all $(p, \chi) \in T_{(\bar{s}, \bar{\phi})} M$ and $u \in[0, t]$,

$$
\left\|v_{u}^{\bar{s}, \bar{\phi}, \chi}\right\|_{C^{1}} \leq c_{2} e^{c_{2} u}\|\chi\|_{C^{1}}
$$


Proof. Proposition 4.1 (i) yields a neighbourhood $V \subset M$ of the compact set $K=$ $\{F(u, s, \phi): 0 \leq u \leq t\}$ in $M$ with

$$
\left.c=\sup _{(\bar{s}, \bar{\phi}) \in V} \| L(\bar{s}, \bar{\phi}, \cdot)\right) \|_{L_{c}(C, \mathbb{R})}<\infty .
$$

A standard compactness argument, which employs the openness of $\Omega$ and the continuity of $F$, shows that there is a neighbourhood $N$ of $(s, \phi)$ in $M$ so that for each $(\bar{s}, \bar{\phi}) \in N$ we have $t<t_{\bar{s}, \bar{\phi}}$ and $F(u, \bar{s}, \bar{\phi}) \in V$ for $0 \leq u \leq t$. Let $(\bar{s}, \bar{\phi}) \in N$ and $(p, \chi) \in T_{(\bar{s}, \bar{\phi})} M, u \in[0, t]$. As in the proof of Proposition 6.1,

$$
\left\|v_{u}^{\bar{s}, \bar{\phi}, \chi}\right\|_{C} \leq e^{c u}\|\chi\|_{C} .
$$

For $a \in[-h, 0]$ with $0<u+a$, Eq. (4.1) gives

$$
\left|\left(v^{\bar{s}, \bar{\phi}, \chi}\right)^{\prime}(u+a)\right| \leq c\left\|v_{u+a}^{\bar{s}, \bar{\phi}, \chi}\right\|_{C} \leq c e^{c u}\|\chi\|_{C} .
$$

As $(p, \chi) \in T_{(\bar{s}, \bar{\phi})} M$ we know from Proposition 4.2 that $v^{\bar{s}, \bar{\phi}, \chi}$ is continuously differentiable. For $a \in[-h, 0]$ with $u+a \leq 0$ this yields

$$
\left|\left(v^{\bar{s}, \bar{\phi}, \chi}\right)^{\prime}(u+a)\right| \leq\|\partial \chi\|_{C} .
$$

Combining the previous estimates we find

$$
\left\|v_{u}^{\bar{s}, \bar{\phi}, \chi}\right\|_{C^{1}} \leq(c+2) e^{c u}\|\chi\|_{C^{1}}
$$

Proposition 6.3. Let $(s, \phi) \in M$ and $h<t<t_{s, \phi}$. Then there exists $c_{3}=$ $c_{3}(t, s, \phi) \geq 0$ so that for every $(p, \chi) \in T_{(s, \phi)} M$ and for $h<u<w \leq t$,

$$
\begin{aligned}
\left\|v_{w}^{s, \phi, \chi}-v_{u}^{s, \phi, \chi}\right\|_{C^{1}} \leq & c_{3}\|\chi\|_{C^{1}}(|w-u|+ \\
& \left.\max _{-h \leq a \leq 0}\left\|L_{1}(F(w+a, s, \phi))-L_{1}(F(u+a, s, \phi))\right\|_{L_{c}\left(C^{1}, \mathbb{R}^{n}\right)}\right) .
\end{aligned}
$$

Proof. 1. Let $(p, \chi) \in T_{(s, \phi)} M, v=v^{s, \phi, \chi}, h<t<t_{s, \phi}$. Estimate of $\left\|v_{w}-v_{u}\right\|_{C}$ for $0 \leq u<w \leq t$ : Proposition 4.2 shows that $v$ is continuously differentiable. By Proposition 4.1 (i),

$$
c=\sup _{0 \leq u \leq t}\|L(F(u, s, \phi), \cdot)\|_{L_{c}\left(C, \mathbb{R}^{n}\right)}<\infty .
$$

Proposition 6.1 yields $c_{1}=c_{1}(t, s, \phi) \geq 0$ with

$$
\left\|v_{u}\right\|_{C} \leq e^{c_{1} t}\|\chi\|_{C} \text { for } 0 \leq u \leq t .
$$

For $0 \leq u<w \leq t$ and for $-h \leq a \leq 0$,

$$
\left|v_{w}(a)-v_{u}(a)\right|=|v(w+a)-v(u+a)|=\left|\int_{u+a}^{w+a} v^{\prime}(y) d y\right| .
$$

In case $-h \leq y \leq 0,\left|v^{\prime}(y)\right|=\left|\chi^{\prime}(y)\right| \leq\|\chi\|_{C^{1}}$. In case $0<y \leq t$,

$$
\left|v^{\prime}(y)\right|=\left|L\left(F(y, s, \phi), v_{y}\right)\right| \leq c\left\|v_{y}\right\|_{C} \leq c e^{c_{1} t}\|\chi\|_{C} .
$$

It follows that for $0 \leq u<w \leq t$,

$$
\left\|v_{w}-v_{u}\right\|_{C} \leq\|\chi\|_{C^{1}}|w-u|\left(1+c e^{c_{1} t}\right) .
$$


2. Next, consider $\left\|\partial v_{w}-\partial v_{u}\right\|_{C}$ for $h<u<w \leq t$. For every $a \in[-h, 0]$,

$$
\begin{aligned}
\left|\partial v_{w}(a)-\partial v_{u}(a)\right|= & \left|v^{\prime}(w+a)-v^{\prime}(u+a)\right| \\
= & \left|L\left(F(w+a, s, \phi), v_{w+a}\right)-L\left(F(u+a, s, \phi), v_{u+a}\right)\right| \\
\leq & \left|L\left(F(w+a, s, \phi), v_{w+a}\right)-L\left(F(u+a, s, \phi), v_{w+a}\right)\right| \\
& +\left|L\left(F(u+a, s, \phi), v_{w+a}-v_{u+a}\right)\right| .
\end{aligned}
$$

Recall the map $L_{1}$ and Proposition 4.1 (ii). As $v_{w+a} \in C^{1}$, the last sum equals $\left|\left(L_{1}(F(w+a, s, \phi))-L_{1}(F(u+a, s, \phi))\right) v_{w+a}\right|+\left|L\left(F(u+a, s, \phi), v_{w+a}-v_{u+a}\right)\right|$ $\leq\left\|L_{1}(F(w+a, s, \phi))-L_{1}(F(u+a, s, \phi))\right\|_{L_{c}\left(C^{1}, \mathbb{R}^{n}\right)}\left\|v_{w+a}\right\|_{C^{1}}+c\left\|v_{w+a}-v_{u+a}\right\|_{C}$. Proposition 6.2 yields $c_{2}=c_{2}(t, s, \phi) \geq 0$ with

$$
\left\|v_{w+a}\right\|_{C^{1}} \leq c_{2} e^{c_{2} t}\|\chi\|_{C^{1}} .
$$

Using this estimate and the result of part 1 we see that the last sum is majorized by

$\left\|L_{1}(F(w+a, s, \phi))-L_{1}(F(u+a, s, \phi))\right\|_{L_{c}\left(C^{1}, \mathbb{R}^{n}\right)} c_{2} e^{c_{2} t}\|\chi\|_{C^{1}}+c\|\chi\|_{C^{1}}|w-u|\left(1+c e^{c_{1} t}\right)$.

It follows that for $h<u<w \leq t$,

$$
\begin{aligned}
\left\|v_{w}-v_{u}\right\|_{C^{1}}= & \left\|v_{w}-v_{u}\right\|_{C}+\left\|\partial v_{w}-\partial v_{u}\right\|_{C} \\
\leq & \|\chi\|_{C^{1}}\left(|s-u|\left(1+c e^{c_{1} t}\right)+\right. \\
& \max { }_{h \leq a \leq 0}\left\|L_{1}(F(w+a, s, \phi))-L_{1}(F(u+a, s, \phi))\right\|_{L_{c}\left(C^{1}, \mathbb{R}^{n}\right)} c_{2} e^{c_{2} t} \\
& \left.+c|w-u|\left(1+c e^{c_{1} t}\right)\right) .
\end{aligned}
$$

Set $c_{3}=c_{2} e^{c_{2} t}+(1+c)\left(1+c e^{c_{1} t}\right)$.

Proposition 6.4. Let $(s, \phi) \in M$ and $h<t<t_{s, \phi}$. There exist $c_{4}=c_{4}(t, s, \phi) \geq 0$ and a neighbourhood $N$ of $(s, \phi)$ in $M$ so that for every $(\bar{s}, \bar{\phi}) \in N$ we have $t<t_{\bar{s}, \bar{\phi}}$, and for all $(p, \chi) \in T_{(\bar{s}, \bar{\phi})} M$ and $u \in(h, t]$,

$\left\|v_{u}^{\bar{s}, \bar{\phi}, \chi}-v_{u}^{s, \phi, \chi}\right\|_{C^{1}} \leq c_{4} e^{c_{4} t} \max _{0 \leq w \leq t}\left\|L_{1}(F(w, \bar{s}, \bar{\phi}))-L_{1}(F(w, s, \phi))\right\|_{L_{c}\left(C^{1}, \mathbb{R}^{n}\right)}\|\chi\|_{C^{1}}$.

Proof. 1. Choose $c_{2}=c_{2}(t, s, \phi)$ and $N$ according to Proposition 6.2 and choose $c$ as in the proof of Proposition 6.1. Let $(\bar{s}, \bar{\phi}) \in N$ and $(p, \chi) \in T_{(\bar{s}, \bar{\phi})} M$. Estimate of $\left\|v_{u}^{\bar{s}, \bar{\phi}, \chi}-v_{u}^{s, \phi, \chi}\right\|_{C}$ for $0 \leq u \leq t$ : Proposition 4.2 shows that $v^{\bar{s}, \bar{\phi}, \chi}$ is continuously differentiable. For $u \in[0, t]$ and $a \in[-h, 0]$ with $u+a \leq 0$,

$$
v^{\bar{s}, \bar{\phi}, \chi}(u+a)-v^{s, \phi, \chi}(u+a)=0 \text {. }
$$

In case $0<u+a$,

$$
\begin{gathered}
\left.\left|v^{\bar{s}, \bar{\phi}, \chi}(u+a)-v^{s, \phi, \chi}(u+a)\right|=\mid \int_{0}^{u+a}\left(v^{\bar{s}, \bar{\phi}, \chi}\right)^{\prime}(y)-\left(v^{s, \phi, \chi}\right)^{\prime}(y)\right) d y \mid \\
=\mid \int_{0}^{u+a}\left(L\left(F(y, \bar{s}, \bar{\phi}), v_{y}^{\bar{s}, \bar{\phi}, \chi}\right)-L\left(F(y, s, \phi), v_{y}^{s, \phi, \chi}\right) d y \mid\right. \\
=\left|\int_{0}^{u+a}\left(L\left(F(y, \bar{s}, \bar{\phi}), v_{y}^{\bar{s}, \bar{\phi}, \chi}\right)-L\left(F(y, s, \phi), v_{y}^{\bar{s}, \bar{\phi}, \chi}\right)\right)+L\left(F(y, s, \phi), v_{y}^{\bar{s}, \bar{\phi}, \chi}-v_{y}^{s, \phi, \chi}\right) d y\right| \\
=\mid \int_{0}^{u+a}\left(L_{1}(F(y, \bar{s}, \bar{\phi}))-L_{1}(F(y, s, \phi)) v_{y}^{\bar{s}, \bar{\phi}, \chi}+L\left(F(y, s, \phi), v_{y}^{\bar{s}, \bar{\phi}, \chi}-v_{y}^{s, \phi, \chi}\right) d y \mid\right.
\end{gathered}
$$


(here we used $v_{y}^{\bar{s}, \bar{\phi}, \chi} \in C^{1}$ for $0 \leq y \leq u+a$ )

$$
\begin{gathered}
\leq t \max _{0 \leq y \leq u+a}\left\|L_{1}(F(y, \bar{s}, \bar{\phi}))-L_{1}(F(y, s, \phi))\right\|_{L_{c}\left(C^{1}, \mathbb{R}^{n}\right)} \max _{0 \leq y \leq u+a}\left\|v_{y}^{\bar{s}, \bar{\phi}, \chi}\right\|_{C^{1}} \\
+c \int_{0}^{u+a}\left\|v_{y}^{\bar{s}, \bar{\phi}, \chi}-v_{y}^{s, \phi, \chi}\right\|_{C} d y \\
\leq t \max _{0 \leq y \leq t}\left\|L_{1}(F(y, \bar{s}, \bar{\phi}))-L_{1}(F(y, s, \phi))\right\|_{L_{c}\left(C^{1}, \mathbb{R}^{n}\right)} c_{2} e^{c_{2}(u+a)}\|\chi\|_{C^{1}} \\
+c \int_{0}^{u+a}\left\|v_{y}^{\bar{s}, \bar{\phi}, \chi}-v_{y}^{s, \phi, \chi}\right\|_{C} d y
\end{gathered}
$$

(see Proposition 6.2). It follows that for $0 \leq u \leq t$,

$\left\|v_{u}^{\bar{s}, \bar{\phi}, \chi}-v_{u}^{s, \phi, \chi}\right\|_{C} \leq t \max _{0 \leq y \leq t}\left\|L_{1}(F(y, \bar{s}, \bar{\phi}))-L_{1}(F(y, s, \phi))\right\|_{L_{c}\left(C^{1}, \mathbb{R}^{n}\right)} c_{2} e^{c_{2} t}\|\chi\|_{C^{1}}$

and Gronwall's lemma yields

$$
+c \int_{0}^{u}\left\|v_{y}^{\bar{s}, \bar{\phi}, \chi}-v_{y}^{s, \phi, \chi}\right\|_{C} d y
$$

$\left\|v_{u}^{\bar{s}, \bar{\phi}, \chi}-v_{u}^{s, \phi, \chi}\right\|_{C} \leq t \max _{0 \leq y \leq t}\left\|L_{1}(F(y, \bar{s}, \bar{\phi}))-L_{1}(F(y, s, \phi))\right\|_{L_{c}\left(C^{1}, \mathbb{R}^{n}\right)} c_{2} e^{c_{2} t}\|\chi\|_{C^{1}} e^{c u}$ for $0 \leq u \leq t$.

2. For $u \in[0, t]$ and $a \in[-h, 0]$ with $0<u+a$,

$$
\begin{aligned}
& \left|\left(v^{\bar{s}, \bar{\phi}, \chi}\right)^{\prime}(u+a)-\left(v^{s, \phi, \chi}\right)^{\prime}(u+a)\right|=\mid L\left(F(u+a, \bar{s}, \bar{\phi}), v_{u+a}^{\bar{s}, \bar{\phi}, \chi}\right)-L\left(F(u+a, s, \phi), v_{u+a}^{s, \phi, \chi} \mid\right. \\
& =\left|L\left(F(u+a, \bar{s}, \bar{\phi}), v_{u+a}^{\bar{s}, \bar{\phi}, \chi}\right)-L\left(F(u+a, s, \phi), v_{u+a}^{\bar{s}, \bar{\phi}, \chi}\right)+L\left(F(u+a, s, \phi), v_{u+a}^{\bar{s}, \bar{\phi}, \chi}-v_{u+a}^{s, \phi, \chi}\right)\right| \\
& \leq \max _{0 \leq y \leq t}\left\|L_{1}(F(y, \bar{s}, \bar{\phi}))-L_{1}(F(y, s, \phi))\right\|_{L_{c}\left(C^{1}, \mathbb{R}^{n}\right)}\left\|v_{u+a}^{\bar{s}, \bar{\phi}, \chi}\right\|_{C^{1}}+c\left\|v_{u+a}^{\bar{s}, \bar{\phi}, \chi}-v_{u+a}^{s, \phi, \chi}\right\|_{C}
\end{aligned}
$$

(compare part 1)

$\leq \max _{0 \leq y \leq t}\left\|L_{1}(F(y, \bar{s}, \bar{\phi}))-L_{1}(F(y, s, \phi))\right\|_{L_{c}\left(C^{1}, \mathbb{R}^{n}\right)} c_{2} e^{c_{2}(u+a)}\|\chi\|_{C^{1}}+c\left\|v_{u+a}^{\bar{s}, \bar{\phi}, \chi}-v_{u+a}^{s, \phi, \chi}\right\|_{C}$

(Proposition 6.2). In case $h<u \leq t$,

$$
v_{u}^{\bar{s}, \bar{\phi}, \chi}-v_{u}^{s, \phi, \chi} \in C^{1} .
$$

Using the previous estimate and the result of part 1 we infer that for $h<u \leq t$,

$$
\begin{gathered}
\left\|\partial v_{u}^{\bar{s}, \bar{\phi}, \chi}-\partial v_{u}^{s, \phi, \chi}\right\|_{C} \\
\leq \max _{0 \leq y \leq t}\left\|L_{1}(F(y, \bar{s}, \bar{\phi}))-L_{1}(F(y, s, \phi))\right\|_{L_{c}\left(C^{1}, \mathbb{R}^{n}\right)}\|\chi\|_{C^{1}}\left(c_{2} e^{c_{2} t}+c t c_{2} e^{\left(c_{2}+c\right) t}\right) .
\end{gathered}
$$

Using the result of part 1 once more we arrive at

$$
\begin{gathered}
\left\|v_{u}^{\bar{s}, \bar{\phi}, \chi}-v_{u}^{s, \phi, \chi}\right\|_{C^{1}} \leq \max _{0 \leq y \leq t}\left\|L_{1}(F(y, \bar{s}, \bar{\phi}))-L_{1}(F(y, s, \phi))\right\|_{L_{c}\left(C^{1}, \mathbb{R}^{n}\right)}\|\chi\|_{C^{1}}\left(t c_{2} e^{\left(c_{2}+c\right) t}\right. \\
\left.+c_{2} e^{c_{2} t}+c t c_{2} e^{\left(c_{2}+c\right) t}\right)
\end{gathered}
$$

for $h<u \leq t,(\bar{s}, \bar{\phi}) \in N,(p, \chi) \in T_{(\bar{s}, \bar{\phi})} M$. 


\section{Derivatives With Respect to initial Data And Smoothness of the SEMIFLOW}

In order to prove that $F$ is continuously differentiable for $t>h$ we need to find charts of the $C^{1}$-submanifold $\mathbb{R} \times M$ of the Banach space $\mathbb{R} \times B$, at points $(t, s, \phi) \in \Omega$ with $t>h$ and with their domains in $\Omega$, so that the composition $\hat{F} \circ K$ with the inverse $K$ of the chart is a continuously differentiable map (from an open subset of a Banach space into $\mathbb{R} \times B$ ). The next result provides a suitable chart.

Proposition 7.1. Let $(t, s, \phi) \in \Omega$ with $t>h$ be given. There exist open neighbourhoods $N$ of $(s, \phi)$ in $M, V$ of 0 in $T_{(s, \phi)} M$, an open interval $I \subset(h, \infty)$ with $t \in I$, and a manifold chart $\alpha: N \rightarrow V$ of $M$ with the following properties.

(i) The continuously differentiable map

$$
\kappa: V \ni(p, \chi) \mapsto \alpha^{-1}(p, \chi) \in B
$$

satisfies $\kappa(0)=(s, \phi)$ and $D \kappa(0)(p, \chi)=(p, \chi)$ on $T_{(s, \phi)} M$.

(ii) $I \times N \subset \Omega$.

(iii) The inverse of the map

$$
K: I \times V \ni(u, p, \chi) \mapsto(u, \kappa(p, \chi)) \in I \times N
$$

is a manifold chart of the submanifold $\mathbb{R} \times M$ of $\mathbb{R} \times B$.

(iv) $\hat{F} \circ K(u, p, \chi)=F(u, \kappa(p, \chi))$ on $I \times V$.

Proof. Choose a complementary closed subspace $Q$ of $T_{(s, \phi)} M$ in $B$. There exist an open neighbourhood $N$ of $(s, \phi)$ in $M$, an open neighbourhood $V$ of 0 in $T_{(s, \phi)} M$, and a continuously differentiable map $\gamma: V \rightarrow Q$ with $\gamma(0)=0, D \gamma(0)=0$, and

$$
N=(s, \phi)+\{(p, \chi)+\gamma(p, \chi) \in B:(p, \chi) \in V\} .
$$

As $\Omega$ is an open subset of $[0, \infty) \times M$ we may assume that for some open interval $I \subset(h, \infty)$ with $t \in I$ we have $I \times N \subset \Omega$. Then the map

$$
\kappa: V \ni(p, \chi) \mapsto(s, \phi)+(p, \chi)+\gamma(p, \chi) \in B
$$

has the properties (i)-(iii).

Choose $(t, s, \phi) \in \Omega$ with $t>h$ and consider $N, V, I, \kappa, K$ according to Proposition 7.1. In the remainder of this section we discuss partial derivatives of $\hat{F} \times K$ : $I \times V \rightarrow B$ with respect to

$$
Y=T_{(s, \phi)} M\left(\subset B=\mathbb{R}^{k} \times C^{1}\right) .
$$

Corollary 7.2. For every $(u, p, \chi) \in I \times V$ the partial derivative

$$
D_{Y}(\hat{F} \circ K)(u, p, \chi): Y \rightarrow B
$$

exists, and for all $(\bar{p}, \bar{\chi}) \in Y$ we have

$$
\begin{aligned}
D_{Y}(\hat{F} \circ K)(u, p, \chi)(\bar{p}, \bar{\chi}) & =\left(q^{\kappa(p, \chi), \hat{\chi}}(u), v_{u}^{\kappa(p, \chi), \hat{\chi}}\right) \\
& =\left(A_{1}(F(u, \kappa(p, \chi))) v_{u}^{\kappa(p, \chi), \hat{\chi}}, v_{u}^{\kappa(p, \chi), \hat{\chi}}\right)
\end{aligned}
$$

with

$$
(\hat{p}, \hat{\chi})=D \kappa(p, \chi)(\bar{p}, \bar{\chi}) .
$$

Proof. Use Proposition 7.1 (iv), Proposition 4.3, and the chain rule. 
The continuity of $D_{Y}(\hat{F} \circ K)(u, p, \chi): Y \rightarrow B$, for $(u, p, \chi) \in I \times V$, implies that the linear maps

$$
p_{1} \circ\left(D_{Y}(\hat{F} \circ K)(u, p, \chi)\right): Y \ni(\bar{p}, \bar{\chi}) \mapsto A_{1}(F(u, \kappa(p, \chi))) v_{u}^{\kappa(p, \chi), \hat{\chi}} \in \mathbb{R}^{k}
$$

and

$$
p_{2} \circ\left(D_{Y}(\hat{F} \circ K)(u, p, \chi)\right): Y \ni(\bar{p}, \bar{\chi}) \mapsto v_{u}^{\kappa(p, \chi), \hat{\chi}} \in C^{1}
$$

with $\hat{\chi}$ given by (7.1) are continuous. Hence

$$
D_{Y}(\hat{F} \circ K)(u, p, \chi)(\bar{p}, \bar{\chi})=(m(u, p, \chi)(\bar{p}, \bar{\chi}), M(u, p, \chi)(\bar{p}, \bar{\chi}))
$$

with the maps $m: I \times V \rightarrow L_{c}\left(Y, \mathbb{R}^{k}\right)$ and $M: I \times V \rightarrow L_{c}\left(Y, C^{1}\right)$ given by

$$
\begin{aligned}
m(u, p, \chi) & =A_{1}(F(u, \kappa(p, \chi))) \circ M(u, p, \chi), \\
M(u, p, \chi)(\bar{p}, \bar{\chi}) & =v_{u}^{\kappa(p, \chi), \hat{\chi}},
\end{aligned}
$$

and (7.1).

The next proposition is the main step towards the continuity of the partial derivative $D_{Y}(\hat{F} \circ K): I \times V \rightarrow L_{c}(Y, B)$. The proof follows the proof of Proposition 8 in [24].

Proposition 7.3. The map $M: I \times V \rightarrow L_{c}\left(Y, C^{1}\right)$ is continuous.

Proof. 1. Fix $u_{0} \in I$ and $\left(p_{0}, \chi_{0}\right) \in V$. Choose $u_{1}>u_{0}$ in $I$ and let $I_{0}=$ $I \cap\left(h, u_{1}\right) \subset(h, \infty)$. Choose a neighbourhood $V_{0}$ of $\left(p_{0}, \chi_{0}\right)$ in $V$ so that

$$
\sup _{(p, \chi) \in V_{0}}\|D \kappa(p, \chi)\|_{L_{c}(Y, B)}<\infty .
$$

For $u \in I_{0},(p, \chi) \in V_{0}$, and $(\bar{p}, \bar{\chi}) \in Y$, we get

$$
\left(M(u, p, \chi)-M\left(u_{0}, p_{0}, \chi_{0}\right)\right)(\bar{p}, \bar{\chi})=G(u, p, \chi, \bar{p}, \bar{\chi})+H(u, \bar{p}, \bar{\chi})
$$

where

with (7.1) and

$$
\begin{aligned}
G(u, p, \chi, \bar{p}, \bar{\chi}) & =v_{u}^{\kappa(p, \chi), \hat{\chi}}-v_{u}^{\kappa\left(p_{0}, \chi_{0}\right), \hat{\chi}_{0}} \\
H(u, \bar{p}, \bar{\chi}) & =v_{u}^{\kappa\left(p_{0}, \chi_{0}\right), \hat{\chi}_{0}}-v_{u_{0}}^{\kappa\left(p_{0}, \chi_{0}\right), \hat{\chi}_{0}}
\end{aligned}
$$

$$
\left(\hat{p}_{0}, \hat{\chi}_{0}\right)=D \kappa\left(p_{0}, \chi_{0}\right)(\bar{p}, \bar{\chi}) .
$$

It remains to show that for $(u, p, \chi) \rightarrow\left(u_{0}, p_{0}, \chi_{0}\right)$ in $I_{0} \times V_{0}$ we have

$$
\|G(u, p, \chi, \bar{p}, \bar{\chi})\|_{C^{1}} \rightarrow 0 \text { and }\|H(u, \bar{p}, \bar{\chi})\|_{C^{1}} \rightarrow 0
$$

uniformly with respect to $(\bar{p}, \bar{\chi})$ in the closed unit ball of the Banach space $Y \subset$ $B=\mathbb{R}^{k} \times C^{1}$.

2. Proposition 6.3 guarantees the existence of $c_{3}=c_{3}\left(u_{1}, \kappa\left(p_{0}, \chi_{0}\right)\right) \geq 0$ so that

$$
\begin{gathered}
\|H(u, \bar{p}, \bar{\chi})\|_{C^{1}} \leq c_{3}\left\|\hat{\chi}_{0}\right\|_{C^{1}}\left(\left|u-u_{0}\right|\right. \\
\left.+\max _{-h \leq a \leq 0}\left\|L_{1}\left(F\left(u+a, \kappa\left(p_{0}, \chi_{0}\right)\right)\right)-L_{1}\left(F\left(u_{0}+a, \kappa\left(p_{0}, \chi_{0}\right)\right)\right)\right\|_{L_{c}\left(C^{1}, \mathbb{R}^{n}\right)}\right)
\end{gathered}
$$

for $u \in I_{0} \subset(h, \infty)$ and $(\bar{p}, \bar{\chi}) \in Y$. Proposition 4.1 (ii) and the continuity of $F$ combined show that the map

$$
\left[0, u_{1}\right] \ni \tilde{u} \mapsto L_{1}\left(F\left(\tilde{u}, \kappa\left(p_{0}, \chi_{0}\right)\right)\right) \in L_{c}\left(C^{1}, \mathbb{R}^{n}\right)
$$

is uniformly continuous. Using this, the previous estimate, and (7.3) one finds easily that for $(u, p, \chi) \rightarrow\left(u_{0}, p_{0}, \chi_{0}\right)$ in $I_{0} \times V_{0}$ we have $\|H(u, \bar{p}, \bar{\chi})\|_{C^{1}} \rightarrow 0$ uniformly 
with respect to $(\bar{p}, \bar{\chi})$ in the closed unit ball of the Banach space $Y \subset B=\mathbb{R}^{k} \times C^{1}$. 3. Choose a neighbourhood $N_{1}$ of $\kappa\left(p_{0}, \chi_{0}\right)$ in $N$ and $c_{4}=c_{4}\left(u_{1}, \kappa\left(p_{0}, \chi_{0}\right)\right) \geq 0$ according to Proposition 6.4. Choose a neighbourhood $V_{1}$ of $\left(p_{0}, \chi_{0}\right)$ in $V_{0}$ so small that $\kappa\left(V_{1}\right) \subset N_{1}$. For all $u \in I_{0},(p, \chi) \in V_{1}$, and $(\bar{p}, \bar{\chi}) \in Y$ we have

$$
\begin{aligned}
G(u, p, \chi, \bar{p}, \bar{\chi})= & v_{u}^{\kappa(p, \chi), \hat{\chi}}-v_{u}^{\kappa\left(p_{0}, \chi_{0}\right), \hat{\chi}_{0}} \\
& (\text { with }(7.1) \text { and }(7.3) \\
= & G_{1}(u, p, \chi, \bar{p}, \bar{\chi})+G_{2}(u, p, \chi, \bar{p}, \bar{\chi})
\end{aligned}
$$

where

$$
\begin{aligned}
& G_{1}(u, p, \chi, \bar{p}, \bar{\chi})=v_{u}^{\kappa(p, \chi), \hat{\chi}}-v_{u}^{\kappa\left(p_{0}, \chi_{0}\right), \hat{\chi}}, \\
& G_{2}(u, p, \chi, \bar{p}, \bar{\chi})=v_{u}^{\kappa\left(p_{0}, \chi_{0}\right), \hat{\chi}}-v_{u}^{\kappa\left(p_{0}, \chi_{0}\right), \hat{\chi}_{0}}=v_{u}^{\kappa\left(p_{0}, \chi_{0}\right), \hat{\chi}-\hat{\chi}_{0} .} .
\end{aligned}
$$

3.1. Proposition 6.4 shows that for $u \in I_{0},(p, \chi) \in V_{1}$, and $(\bar{p}, \bar{\chi}) \in Y$, and for $\hat{\chi}$ given by (7.1) we have

$$
\begin{gathered}
\left\|G_{1}(u, p, \chi, \bar{p}, \bar{\chi})\right\|_{C^{1}} \\
\leq c_{4} e^{c_{4} u_{1}} \max _{0 \leq \tilde{u} \leq u_{1}}\left\|L_{1}(F(\tilde{u}, \kappa(p, \chi)))-L_{1}\left(F\left(\tilde{u}, \kappa\left(p_{0}, \chi_{0}\right)\right)\right)\right\|_{L_{c}\left(C^{1}, \mathbb{R}^{n}\right)}\|\hat{\chi}\|_{C^{1}} .
\end{gathered}
$$

By a standard continuity and compactness argument,

$$
\max _{0 \leq \tilde{u} \leq u_{1}}\left\|L_{1}(F(\tilde{u}, \kappa(p, \chi)))-L_{1}\left(F\left(\tilde{u}, \kappa\left(p_{0}, \chi_{0}\right)\right)\right)\right\|_{L_{c}\left(C^{1}, \mathbb{R}^{n}\right)} \rightarrow 0
$$

as $(p, \chi) \rightarrow\left(p_{0}, \chi_{0}\right)$ in $V_{1} \subset Y$. Using this statement, the previous estimate, and the boundedness of $\|D \kappa(p, \chi)\|$ on $V_{0}$ we infer

$$
\left\|G_{1}(u, p, \chi, \bar{p}, \bar{\chi})\right\|_{C^{1}} \rightarrow 0
$$

as $(u, p, \chi) \rightarrow\left(u_{0}, p_{0}, \chi_{0}\right)$ in $I_{0} \times V_{1} \subset \mathbb{R} \times Y$, uniformly for $(\bar{p}, \bar{\chi})$ in the closed unit ball of the Banach space $Y \subset B=\mathbb{R}^{k} \times C^{1}$.

3.2. Finally, Proposition 6.1 yields $c_{1}=c_{1}\left(u_{1}, \kappa\left(p_{0}, \chi_{0}\right)\right) \geq 0$ so that for $u \in I_{0}$, $(p, \chi) \in V_{1}$, and $(\bar{p}, \bar{\chi}) \in Y$, and for $\hat{\chi}$ given by (7.1) we have

$$
\begin{gathered}
\left\|G_{2}(u, p, \chi, \bar{p}, \bar{\chi})\right\|_{C^{1}} \leq c_{1} e^{c_{1} u_{1}}\left\|\hat{\chi}-\hat{\chi}_{0}\right\|_{C^{1}} \\
\leq c_{1} e^{c_{1} u_{1}}\left\|D \kappa(p, \chi)-D \kappa\left(p_{0}, \chi_{0}\right)\right\|_{L_{c}(Y, B)}\|(\bar{p}, \bar{\chi})\|_{B} .
\end{gathered}
$$

Now it is obvious how to complete the proof.

Corollary 7.4. The map $m: I \times V \rightarrow L_{c}\left(Y, \mathbb{R}^{k}\right)$ is continuous.

Proof. Use the definition of $m$, the continuity of $F, k$ and $A_{1}$, and Proposition 7.3 .

Corollary 7.5. The map $D_{Y}(\hat{F} \circ K): I \times V \rightarrow L_{c}(Y, B)$ is continuous.

Proof. For $(u, p, \chi)$ and $\left(u_{0}, p_{0}, \chi_{0}\right)$ in $I \times V$ and $(\bar{p}, \bar{\chi}) \in Y$ with $\|(\bar{p}, \bar{\chi})\|_{B} \leq 1$ we infer from Eq. (7.2) that

$$
\begin{gathered}
\left\|\left(D_{Y}(\hat{F} \circ K)(u, p, \chi)-D_{Y}(\hat{F} \circ K)\left(u_{0}, p_{0}, \chi_{0}\right)\right)(\bar{p}, \bar{\chi})\right\|_{B} \\
=\mid\left(m(u, p, \chi)-m\left(u_{0}, p_{0}, \chi_{0}\right)(\bar{p}, \bar{\chi}) \mid+\|\left(M(u, p, \chi)-M\left(u_{0}, p_{0}, \chi_{0}\right)(\bar{p}, \bar{\chi}) \|_{C^{1}}\right.\right. \\
\leq\left|m(u, p, \chi)-m\left(u_{0}, p_{0}, \chi_{0}\right)\right|_{L_{c}\left(Y, \mathbb{R}^{k}\right)}+\left\|M(u, p, \chi)-M\left(u_{0}, p_{0}, \chi_{0}\right)\right\|_{L_{c}\left(Y, C^{1}\right)} .
\end{gathered}
$$

Use this, Proposition 7.3, and Corollary 7.4.

Theorem 7.6. The restriction of $F$ to the open subset $\{(t, s, \phi) \in \Omega: t>h\} \neq \emptyset$ of the continuosly differentiable submanifold $\mathbb{R} \times M$ of the Banach space $\mathbb{R} \times B=$ $\mathbb{R} \times \mathbb{R}^{k} \times C^{1}$ is continuously differentiable. 
Proof. Let $(t, s, \phi) \in \Omega$ with $t>h$ be given. Consider $N, V, I, \kappa, K$ as in Proposition 7.1, and $Y=T_{(s, \phi)} M$. We need to show that $\hat{F} \circ K: I \times V \rightarrow B$ has continuously differentiable partial derivatives with respect to the components of $\mathbb{R} \times Y$. Corollary 7.5 takes care of $D_{Y}(\hat{F} \circ K)$. From Proposition 7.1 (iv) we infer

$$
D_{1}(\hat{F} \circ K)(u, p, \chi) 1=D \hat{F}_{\kappa(p, \chi)}(u) 1
$$

for all $(u, p, \chi) \in I \times V$. This formula, Proposition 5.1(iii), and the continuity of $\kappa$ combined yield that $D_{1}(\hat{F} \circ K): I \times V \rightarrow L_{c}(\mathbb{R}, B)$ is continuous. 


\section{A MODEl EQUATION AND TEMPORAL ORDER OF REACTIONS}

In this section we study the equations from section 1 which model the regulation of the density of white blood cells, but shall not make use of the monotonicity properties of the delay function $d$ and of the production function $f$ in the model. So let $h>0, \mu \geq 0$ and continuously differentiable functions $f: \mathbb{R} \rightarrow \mathbb{R}$ and $d: \mathbb{R} \rightarrow(0, h)$ be given. For $n=1=k$ and $U=(-h, 0) \times C^{1}$ we consider the maps

$$
g: U \rightarrow \mathbb{R} \text { and } \Delta: U \rightarrow \mathbb{R}
$$

defined by

$$
\begin{aligned}
g(s, \phi) & =g_{\mu, f}(s, \phi)=-\mu \phi(0)+f(\phi(s)), \\
\Delta(s, \phi) & =\Delta_{d}(s, \phi)=s+d(\phi(s))
\end{aligned}
$$

and verify hypothesis $(\mathrm{H})$ : Both maps $g_{\mu, f}=-\mu e v_{1,0} \circ p r_{2}+f \circ e v_{1}$ and $\Delta_{d}=$ $p r_{1}+d \circ e v_{1}$ are continuously differentiable. For $(s, \phi) \in(-h, 0) \times C^{1}$ and $(p, \chi) \in B$ (with $k=1$ ) we have

$$
D g_{\mu, f}(s, \phi)(p, \chi)=-\mu \chi(0)+f^{\prime}(\phi(s))\left[p \phi^{\prime}(s)+\chi(s)\right] .
$$

The previous formula defines extensions $D_{e} g_{\mu, f}(s, \phi) \in L_{c}(\mathbb{R} \times C, \mathbb{R})$, and the map

$$
\Lambda_{\mu, f}:(-h, 0) \times C^{1} \times \mathbb{R} \times C \ni(s, \phi, p, \chi) \mapsto D_{e} g_{\mu, f}(s, \phi)(p, \chi) \in \mathbb{R}
$$

is continuous (Here we use that $e v_{0}$ and $\partial$ are continuous). For $\Delta_{d},(s, \phi) \in(-h, 0) \times$ $C^{1}$ and $(p, \chi) \in B$ we have

$$
\begin{aligned}
D_{1} \Delta_{d}(s, \phi) 1 & =d^{\prime}(\phi(s)) \phi^{\prime}(s)+1 \\
D_{2} \Delta_{d}(s, \phi) \chi & =d^{\prime}(\phi(s)) \chi(s) .
\end{aligned}
$$

The last formula defines a linear extension $D_{2, e} \Delta_{d}(s, \phi): C \rightarrow \mathbb{R}$ of $D_{2} \Delta(s, \phi) \in$ $L_{c}\left(C^{1}, \mathbb{R}\right)$ which is continuous as it is given by $d^{\prime}\left(e v_{1}(s, \phi)\right) e v_{0}(s, \chi)$. We also see that the map

$$
(-h, 0) \times C^{1} \times C \ni(s, \phi, \chi) \mapsto D_{2, e} \Delta_{d}(s, \phi) \chi \in \mathbb{R}
$$

is continuous.

We obtain

$$
\begin{aligned}
M= & \left\{(s, \phi) \in(-h, 0) \times C^{1}: \phi^{\prime}(0)=-\mu \phi(0)+f(\phi(s)), 0=d(\phi(s))+s,\right. \\
& \left.0 \neq d^{\prime}(\phi(s)) \phi^{\prime}(s)+1\right\}
\end{aligned}
$$

and the initial value problem (1.2)-(1.4) becomes

$$
\begin{aligned}
x^{\prime}(t) & =-\mu x(t)+f(x(t+r(t)) \text { for } t>0, \\
0 & =d(x(t+r(t)))+r(t) \text { for } t>0, \\
\left(r(0), x_{0}\right) & =(s, \phi) .
\end{aligned}
$$

Also,

$$
A(s, \phi, \chi)=-\frac{d^{\prime}(\phi(s)) \chi(s)}{d^{\prime}(\phi(s)) \phi^{\prime}(s)+1}
$$

for $(s, \phi) \in M$ and $\chi \in C$, and the equation in Proposition 3.4 becomes

$$
r^{\prime}(t)=-\frac{d^{\prime}(x(t+r(t))) x^{\prime}(t+r(t))}{d^{\prime}(x(t+r(t))) x^{\prime}(t+r(t))+1} .
$$


The equation in part (i) of the subsequent proposition may be convenient for later use. Part (ii) implies that the set of all $\phi \in C^{1}$ with det $D_{1} \Delta(s, \phi)>0$ for some $s \in(-h, 0)$ is open and dense in $C^{1}$.

Proposition 8.1. (i) Suppose $(r, x)$ is a solution of the system (8.1)-(8.2) with domain $\left[0, t_{e}\right)$, and $t=s+r(s)$ for some $s \in\left[0, t_{e}\right)$. Then

$$
t+d(x(t)) \in\left[0, t_{e}\right) \quad \text { and } \quad r(t+d(x(t)))=-d(x(t)) .
$$

(ii) For every $\phi \in C^{1}$ there exists $s \in-d(\mathbb{R}) \subset(-h, 0)$ with

$$
\Delta(s, \phi)=d(\phi(s))+s=0 \quad \text { and } \quad \operatorname{det} D_{1} \Delta(s, \phi) 1=(d \circ \phi)^{\prime}(s)+1 \geq 0 .
$$

Proof. Assertion (i) follows from

$$
\begin{aligned}
-d(x(t)) & =-d(x(s+r(s)))=-d\left(x_{s}(r(s))\right) \\
& =r(s) \text { and } \\
s & =t-r(s))=t+d\left(x_{s}(r(s))\right)=t+d(x(s+r(s)))=t+d(x(t)) .
\end{aligned}
$$

Assertion (ii) follows from $(d \circ \phi)(-h)-h<0<(d \circ \phi)(0)+0$ and continuity.

In the sequel we discuss the monotonicity properties of the delayed argument functions

$$
\tau^{s, \phi}:\left[0, t_{s, \phi}\right) \ni t \mapsto t+r^{s, \phi}(t) \in \mathbb{R}
$$

for $(s, \phi) \in M$. These functions are continuously differentiable and satisfy

$$
-h<\tau^{s, \phi}(t)<t_{s, \phi} \text { for all } t \in\left[0, t_{s, \phi}\right) .
$$

Notice that for $x=x^{s, \phi}$ and $\tau=\tau^{s, \phi}$, with $(s, \phi) \in M$, Eq. (8.1) reads

$$
x^{\prime}(t)=-\mu x(t)+f(x(\tau(t))) .
$$

The manifold $M$ is the disjoint union of its open subsets

$$
M_{ \pm}=\left\{(s, \phi) \in M: \operatorname{det} D_{1} \Delta(s, \phi) \gtrless 0\right\} .
$$

Proposition 8.2. For every $(s, \phi) \in M_{ \pm}$and for all $t \in\left[0, t_{s, \phi}\right)$,

$$
F(t, s, \phi) \in M_{ \pm} \quad \text { and } \quad\left(\tau^{s, \phi}\right)^{\prime}(t) \gtreqless 0 .
$$

For any flowline $(r, X): I \rightarrow M$,

$$
\text { either }(r(t), X(t)) \in M_{+} \quad \text { on } \quad I \quad \text { or } \quad(r(t), X(t)) \in M_{-} \quad \text { on } \quad I .
$$

Proof. Let $(s, \phi) \in M, r=r^{s, \phi}, x=x^{s, \phi}$. The first assertion follows since Proposition 3.4 and Eq. (8.4) combined yield

$$
\left(\tau^{s, \phi}\right)^{\prime}(t)=1+r^{\prime}(t)=\frac{1}{d^{\prime}(x(t+r(t))) x^{\prime}(t+r(t))+1}=\frac{1}{D_{1} \Delta\left(r(t), x_{t}\right) 1}
$$

on $\left[0, t_{s, \phi}\right)$. The second assertion is a consequence of the first one.

In light of the preceding result Eq. (8.1) for the evolution in time of the state $x(t)$ of the system under consideration says the following. If a flowline $F(\cdot, s, \phi)$ starts from $(s, \phi) \in M_{+}$then for $x=x^{s, \phi}$ and $\tau=\tau^{s, \phi}$ we have that adjustments $x^{\prime}\left(t_{1}\right)$ and $x^{\prime}\left(t_{2}\right)$ to previous states $x\left(\tau\left(t_{1}\right)\right)$ and $x\left(\tau\left(t_{2}\right)\right)$, with $\tau\left(t_{1}\right)<\tau\left(t_{2}\right)$, occur always in the same temporal order, i. e., at $t_{1}<t_{2}$. On the other hand, initial data in $M_{-}$result in adjustments of the state always in reverse temporal order. Such flowlines with decreasing delayed argument function are short-lived, according to 
the following observation.

Corollary 8.3. For every $(s, \phi) \in M_{-}, t_{s, \phi} \leq h$.

Proof. From $t-h<t+r^{s, \phi}(t)=\tau^{s, \phi}(t) \leq \tau^{s, \phi}(0)=r^{s, \phi}(0)<0$ for $0 \leq t<t_{s, \phi}$, $t_{s, \phi} \leq h$.

Next we show that in case $f$ is bounded each flowline $F(\cdot, s, \phi),(s, \phi) \in M$, with $t_{s, \phi}<\infty$ has a limit at $t_{s, \phi}$. We begin with bounds for solutions. Recall first that

$$
-h<r^{s, \phi}(t)<0 \quad \text { for all } \quad(s, \phi) \in M \quad \text { and } t \in\left[0, t_{s, \phi}\right),
$$

due to Eq. (8.2) and the hypothesis $d(\mathbb{R}) \subset(0, h)$.

Proposition 8.4. Suppose $c=\sup _{\xi \in \mathbb{R}}|f(\xi)|<\infty$. Then each function $x^{s, \phi}$, $(s, \phi) \in M$, is bounded. In case $|\phi(0)| \leq \frac{c}{\mu},\left|x^{s, \phi}(t)\right| \leq \frac{c}{\mu}$ for all $t \in\left[0, t_{s, \phi}\right)$. If $\phi(0)>\frac{c}{\mu}$ then $-\frac{c}{\mu} \leq x^{s, \phi}(t) \leq \phi(0)$ for all $t \in\left[0, t_{s, \phi}\right)$. If $\phi(0)<-\frac{c}{\mu}$ then $\phi(0) \leq x^{s, \phi}(t) \leq \frac{c}{\mu}$ for all $t \in\left[0, t_{s, \phi}\right)$.

Proof. Let $(s, \phi) \in M, x=x^{s, \phi}$. On $\left(0, t_{s, \phi}\right)$ we have

$$
-\mu\left(x(t)+\frac{c}{\mu}\right) \leq x^{\prime}(t)=\frac{d}{d t}\left(u \mapsto x(u) \pm \frac{c}{\mu}\right)(t) \leq-\mu\left(x(t)-\frac{c}{\mu}\right) .
$$

Hence

$$
x(t)-\frac{c}{\mu} \leq\left(x(0)-\frac{c}{\mu}\right) e^{-\mu t}
$$

and

$$
\left(x(0)+\frac{c}{\mu}\right) e^{-\mu t} \leq x(t)+\frac{c}{\mu}
$$

on $\left[0, t_{e}\right)$. These inequalities yield the assertions.

Proposition 8.5. Suppose $c=\sup _{\xi \in \mathbb{R}}|f(\xi)|<\infty$ and let $(s, \phi) \in M$. In case $t_{s, \phi}<\infty$ there exists $(w, \psi) \in(-h, 0) \times C^{1} \subset B$ so that

$$
\begin{aligned}
\lim _{t \nearrow t_{s, \phi}}\left(r^{s, \phi}(t), x_{t}^{s, \phi}\right) & =(w, \psi), \\
\psi^{\prime}(0) & =g(w, \psi)=-\mu \psi(0)+f(\psi(w)), \\
0 & =\Delta(w, \psi)=d(\psi(w))+w, \\
0 & =\operatorname{det} D_{1} \Delta(w, \psi)=d^{\prime}(\psi(w)) \psi^{\prime}(w)+1 .
\end{aligned}
$$

In particular, $(w, \psi) \in \bar{M} \backslash M$.

Proof. Let $(s, \phi) \in M, r=r^{s, \phi}, x=x^{s, \phi}, t_{e}=t_{s, \phi}, \tau=\tau^{s, \phi} . \tau$ is bounded (by $h+t_{e}$ ) and monotone (Proposition 8.2). Therefore it has a limit at $t_{e}$. It follows that also $r:\left[0, t_{e}\right) \ni t \rightarrow \tau(t)-t \in[-h, 0]$ has a limit $w \in[-h, 0]$ at $t_{e}$. The boundedness of $x$ and Eq. (8.1) combined yield that $x^{\prime}$ is bounded. It follows that for $t \nearrow t_{e}$ we have

$$
x(t) \rightarrow x(0)+\int_{\left(0, t_{e}\right)} x^{\prime}(u) d u=\xi \in \mathbb{R} .
$$


Define $\psi:[-h, 0] \rightarrow \mathbb{R}$ by $\psi(a)=x\left(t_{e}+a\right)$ for $-h \leq a<0$ and $\psi(0)=\xi$. Then $\psi \in C$ and $\left\|x_{t}-\psi\right\|_{C} \rightarrow 0$ as $t \nearrow t_{e}$. As $e v_{0}$ and $f$ are continuous we infer that

$$
x^{\prime}(t)=-\mu x(t)+\left(f \circ e v_{0}\right)\left(r(t), x_{t}\right) \rightarrow-\mu \psi(0)+f(\psi(w)) \text { as } t \nearrow t_{e} .
$$

We conclude that $\psi \in C^{1}$,

$$
\psi^{\prime}(0)=-\mu \psi(0)+f(\psi(w)),
$$

and $\left\|x_{t}-\psi\right\|_{C^{1}} \rightarrow 0$ as $t \nearrow t_{e}$. It follows that $\left\|\left(r(t), x_{t}\right)-(w, \psi)\right\|_{B} \rightarrow 0$ as $t \nearrow t_{e}$. Next,

$$
0=\lim _{t>t_{e}}\left(d \circ e v_{0}\right)\left(r(t), x_{t}\right)+r(t)=\left(d \circ e v_{0}\right)(w, \psi)+w=d(\psi(w))+w .
$$

As $d$ has range in $(0, h)$ we infer $-h<w<0$. Finally, suppose $d^{\prime}(\psi(w)) \psi^{\prime}(w)+1 \neq$ 0 . Then det $D_{1} \Delta(w, \psi)=D_{1} \Delta(w, \psi) 1 \neq 0,(w, \psi) \in M$, and we can use the flowline $F(\cdot, w, \psi)$ in order to obtain a contradiction to the fact that $(r, x)$ with domain $\left[0, t_{e}\right)$ is a maximal solution.

The preceding proposition suggests to ask for continuation of flowlines $F(\cdot, s, \phi)$ beyond $t_{s, \phi}<\infty$, using the limiting state $(w, \psi) \in B$ as a new initial condition. Moreover, would it be possible that a flowline with, say, increasing delayed argument can be continued beyond $t_{s, \phi}<\infty$ by a flowline with decreasing delayed argument, and vice versa? The following result excludes such transient behaviour.

Proposition 8.6. Suppose $(x, r)$ is a solution of the system (8.1)-(8.2) with domain $\left[0, t_{e}\right), 0<t_{e} \leq \infty$, and there exists $t_{0} \in\left(0, t_{e}\right)$ with

$$
\left(r(t), x_{t}\right) \in M \quad \text { for all } t \in\left[0, t_{e}\right) \backslash\left\{t_{0}\right\} .
$$

Then either $\left(r(t), x_{t}\right) \in M_{-}$for all $t \in\left[0, t_{e}\right) \backslash\left\{t_{0}\right\}$, or $\left(r(t), x_{t}\right) \in M_{+}$for all $t \in\left[0, t_{e}\right) \backslash\left\{t_{0}\right\}$.

Proof. 1. The maps $\left[0, t_{0}\right) \ni t \mapsto\left(r(t), x_{t}\right) \in M$ and $\left(t_{0}, t_{e}\right) \ni t \mapsto\left(r(t), x_{t}\right) \in M$ are flowlines. Therefore Proposition 8.2 yields that the sign of det $D_{1} \Delta\left(r(t), x_{t}\right) \neq 0$ is constant on each of the intervals $\left[0, t_{0}\right)$ and $\left(t_{0}, t_{e}\right)$.

2. Set $\phi=x_{t_{0}} \in C^{1}$. By continuity and $-h<r\left(t_{0}\right)<0$, there exists $\delta>0$ so that $0<t_{0}-\delta, t_{0}+\delta<t_{e}$ and $-h<r\left(t_{0}+u\right)+u<0$ on $(-\delta, \delta)$. For the continuous maps

$$
\rho:(-\delta, \delta) \ni u \mapsto r\left(t_{0}+u\right) \in \mathbb{R} \quad \text { and } \quad \tau:(-\delta, \delta) \ni u \mapsto \rho(u)+u \in(-h, 0) .
$$

we have

$$
\begin{aligned}
0= & \Delta\left(r\left(t_{0}+u\right), x_{t_{0}+u}\right)=d\left(x\left(t_{0}+u+r\left(t_{0}+u\right)\right)\right)+r\left(t_{0}+u\right) \\
= & (d \circ \phi)(\rho(u)+u)+\rho(u) \\
= & (d \circ \phi)(\tau(u))+\tau(u)-u \text { on }(-\delta, \delta), \\
& \quad \text { and for } 0<|u|<\delta, \\
0 \neq & \operatorname{det} D_{1} \Delta\left(r\left(t_{0}+u\right), x_{t_{0}+u}\right)=d^{\prime}\left(x\left(t_{0}+u+r\left(t_{0}+u\right)\right)\right) x^{\prime}\left(t_{0}+u+r\left(t_{0}+u\right)\right)+1 \\
= & d^{\prime}\left(x\left(t_{0}+u+\rho(u)\right)\right) x^{\prime}\left(t_{0}+u+\rho(u)\right)+1 \\
= & d^{\prime}\left(x\left(t_{0}+\tau(u)\right) x^{\prime}\left(t_{0}+\tau(u)\right)+1=d^{\prime}\left(x_{t_{0}}(\tau(u))\left(x_{t_{0}}\right)^{\prime}(\tau(u))+1\right.\right. \\
= & d^{\prime}(\phi(\tau(u))) \phi^{\prime}(\tau(u))+1=(d \circ \phi)^{\prime}(\tau(u))+1 .
\end{aligned}
$$


Using the Implicit Function Theorem and continuity we infer that $\tau$ is differentiable for $0<|u|<\delta$. From the equation $0=d \circ \phi \circ \tau(u)+\tau(u)-u$ we obtain

$$
\tau^{\prime}(u)=\frac{1}{(d \circ \phi)^{\prime}(\tau(u))+1} \quad \text { for } \quad 0<|u|<\delta .
$$

3. Suppose now the assertion is false. It follows that there exists $j \in\{0,1\}$ so that for all $u \in(-\delta, 0)$ and for all $w \in(0, \delta)$ we have

$$
\operatorname{sign}\left(\operatorname{det} D_{1} \Delta\left(r\left(t_{0}+u\right), x_{t_{0}+u}\right)\right)=(-1)^{j}=-\operatorname{sign}\left(\operatorname{det} D_{1} \Delta\left(r\left(t_{0}+w\right), x_{t_{0}+w}\right)\right),
$$

or equivalently,

$$
\operatorname{sign}\left((d \circ \phi)^{\prime}(\tau(u))+1\right)=(-1)^{j}=-\operatorname{sign}\left((d \circ \phi)^{\prime}(\tau(w))+1\right) .
$$

Using the equations (8.7)-(8.8) and continuity we infer that at $u=0 \tau$ has a strict local extremum. It follows that there exist $u_{*} \in(-\delta, 0)$ and $w_{*} \in(0, \delta)$ with $\tau\left(u_{*}\right)=\tau\left(w_{*}\right)$, which yields a contradiction to Eq. (8.8) with $u=u_{*}$ and $w=w_{*}$.

Next we find initial data $(s, \phi) \in \bar{M} \backslash M$ from which two flowlines bifurcate, one into $M_{+}$and the other one into $M_{-}$. This is, of course, a case of nonuniqueness for the initial value problem (8.1)-(8.3).

Proposition 8.7. Suppose $d$ is not constant. Then there exist $(s, \phi) \in \bar{M} \backslash M$, $t_{e}>0$ and solutions $\left(r^{ \pm}, x^{ \pm}\right)$of the initial value problem (8.1)-(8.3) with common domain $\left[0, t_{e}\right)$ so that

$$
\left(r^{ \pm}(t), x_{t}^{ \pm}\right) \in M_{ \pm} \quad \text { on } \quad\left(0, t_{e}\right)
$$

with the delayed argument function $\tau^{+}:\left[0, t_{e}\right) \ni t \mapsto t+r^{+}(t) \in \mathbb{R}$ strictly increasing and the delayed argument function $\tau^{-}:\left[0, t_{e}\right) \ni t \mapsto t+r^{-}(t) \in \mathbb{R}$ strictly decreasing.

Proof. Choose $\xi \in \mathbb{R}$ with $d^{\prime}(\xi) \neq 0$. Set $s=-d(\xi) \in-d(\mathbb{R}) \subset(-h, 0)$. The function $d$ maps an open interval $I \ni \xi$ one-to-one onto an open interval $J \ni-s$. Choose an open interval $Z \subset(-h, 0)$ with $s \in Z$ so that for all $z \in Z$ we have $(z-s)^{2}-z \in J$. Then there exists $\phi \in C^{1}$ with

$$
(d \circ \phi)(z)=(z-s)^{2}-z \text { for all } z \in Z
$$

and

Choose $t_{e}>0$ with

$$
\phi^{\prime}(0)=-\mu \phi(0)+f(\phi(s)) .
$$

$$
s \pm \sqrt{t_{e}} \in Z
$$

and

$$
-h<s-\sqrt{t_{e}}-t_{e} .
$$

Define two functions $\tau^{ \pm}:\left[0, t_{e}\right) \rightarrow \mathbb{R}$ by $\tau^{ \pm}(t)=s \pm \sqrt{t}$. The continuous functions

$$
r^{ \pm}:\left[0, t_{e}\right) \ni t \mapsto \tau^{ \pm}(t)-t \in \mathbb{R}
$$

satisfy $-h<r^{ \pm}(t)<0$ for all $t \in\left[0, t_{e}\right)$. Define $x^{ \pm}:\left[-h, t_{e}\right) \rightarrow \mathbb{R}$ by $x_{0}=\phi$ and

$$
x^{ \pm}(t)=e^{-\mu t} \phi(0)+\int_{0}^{t} e^{-\mu(t-u)} f\left(\phi\left(\tau^{ \pm}(u)\right)\right) d u
$$


for $0<t<t_{e}$. These functions are continuously differentiable and satisfy

$$
\left(x^{ \pm}\right)^{\prime}(t)=-\mu x^{ \pm}(t)+f\left(x^{ \pm}\left(\tau^{ \pm}(t)\right)\right)=-\mu x^{ \pm}(t)+f\left(x^{ \pm}\left(t+r^{ \pm}(t)\right)\right)
$$

for $0 \leq t<t_{e}$. For such $t$ we also have

$$
\begin{aligned}
\Delta\left(r^{ \pm}(t), x_{t}^{ \pm}\right) & =d\left(x_{t}^{ \pm}\left(r^{ \pm}(t)\right)\right)+r^{ \pm}(t) \\
& =d\left(x^{ \pm}\left(t+r^{ \pm}(t)\right)\right)+\tau^{ \pm}(t)-t \\
& =d\left(\phi\left(\tau^{ \pm}(t)\right)\right)+\tau^{ \pm}(t)-t \\
& =\left(\tau^{ \pm}(t)-s\right)^{2}-\tau^{ \pm}(t)+\tau^{ \pm}(t)-t=0,
\end{aligned}
$$

and for $0<t<t_{e}$,

$$
\begin{aligned}
\operatorname{det} D_{1} \Delta\left(r^{ \pm}(t), x_{t}^{ \pm}\right) & =d^{\prime}\left(x_{t}^{ \pm}\left(r^{ \pm}(t)\right)\right)\left(x_{t}^{ \pm}\right)^{\prime}\left(r^{ \pm}(t)\right)+1 \\
& =d^{\prime}\left(x^{ \pm}\left(t+r^{ \pm}(t)\right)\right)\left(x^{ \pm}\right)^{\prime}\left(t+r^{ \pm}(t)\right)+1 \\
& =d^{\prime}\left(\phi\left(\tau^{ \pm}(t)\right)\right) \phi^{\prime}\left(\tau^{ \pm}(t)\right)+1 \\
& =(d \circ \phi)^{\prime}\left(\tau^{ \pm}(t)\right)+1=2\left(\tau^{ \pm}(t)-s\right)-1+1= \pm 2 \sqrt{t} \neq 0 .
\end{aligned}
$$

Altogether, the pairs $\left(r^{ \pm}, x^{ \pm}\right)$are solutions of the initial value problem (8.1)-(8.3) with common domain $\left[0, t_{e}\right)$ and satisfy

$$
\begin{aligned}
\left(r^{ \pm}(t), x_{t}^{ \pm}\right) & \in M_{ \pm} \text {on }\left(0, t_{e}\right), \\
\left(r^{ \pm}(0), x_{0}^{ \pm}\right) & =(s, \phi) \in(-h, 0) \times C^{1}, \\
\left(\tau^{+}\right)^{\prime}(t) & =\frac{1}{2 \sqrt{t}}>0 \text { on }\left(0, t_{e}\right), \\
\left(\tau^{-}\right)^{\prime}(t) & =-\frac{1}{2 \sqrt{t}}<0 \text { on }\left(0, t_{e}\right) .
\end{aligned}
$$

In particular, $r^{+}(t) \neq r^{-}(t)$ on $\left(0, t_{e}\right)$. The curves $\left[0, t_{e}\right) \ni t \mapsto\left(r^{ \pm}(t), x_{t}^{ \pm}\right) \in$ $\mathbb{R} \times C^{1}=B$ are continuous, hence $(s, \phi)=\lim _{t \searrow_{0}}\left(r^{ \pm}(t), x_{t}^{ \pm}\right) \in \bar{M}$. We have $(s, \phi) \notin M$ because of nonuniqueness (or, because of

$$
\left.\operatorname{det} D_{1} \Delta(s, \phi)=2\left(\tau^{ \pm}(0)-s\right)=0\right) \text {. }
$$

We turn to termination of flowlines and show that a pair of flowlines, one with increasing delayed argument and the other one with decreasing delayed argument, terminate at the same argument $t_{e}<\infty$, with the same delayed argument.

Proposition 8.8. Suppose $d$ is not constant. Then there exist $\phi^{ \pm} \in C^{1}, s^{+}<w<$ $s^{-}$in $(-h, 0), t_{e} \in(0, \infty)$ and $\psi^{ \pm} \in C^{1}$ with the following properties: $\left(s^{ \pm}, \phi^{ \pm}\right) \in$ $M_{ \pm}, t_{s^{-}, \phi^{-}}=t_{e}=t_{s^{+}, \phi^{+}}$

$$
\begin{aligned}
& \lim _{t \nearrow t_{e}} F\left(t, s^{-}, \phi^{-}\right)=\left(w, \psi^{-}\right), \\
& \lim _{t \nearrow t_{e}} F\left(t, s^{+}, \phi^{+}\right)=\left(w, \psi^{+}\right) .
\end{aligned}
$$

Proof. Choose $\xi \in \mathbb{R}$ with $d^{\prime}(\xi) \neq 0$. Set $s=-d(\xi) . d$ maps an open interval $I \ni \xi$ one-to-one onto an open interval $J \ni-s$. Choose $t_{e}>0$ and an open interval 
$Z \subset(-h, 0)$ with $s \in Z$ and

$$
\begin{aligned}
t_{e}-(z-s)^{2}-z & \in J \text { for all } z \in Z, \\
s \pm \sqrt{t_{e}} & \in Z, \\
s-\sqrt{t_{e}}-t_{e} & >-h .
\end{aligned}
$$

Define $\tau^{ \pm}:\left[0, t_{e}\right] \rightarrow \mathbb{R}$ by

$$
\tau^{ \pm}(t)=s \mp \sqrt{t_{e}-t} .
$$

Then $\tau^{ \pm}(t) \in Z \subset(-h, 0)$ for all $t \in\left[0, t_{e}\right]$, and the continuous function

$$
r^{ \pm}:\left[0, t_{e}\right] \ni t \mapsto \tau^{ \pm}(t)-t \in \mathbb{R}
$$

satisfies $0>r^{ \pm}(t) \geq s-\sqrt{t_{e}}-t_{e}>-h$ on $\left[0, t_{e}\right]$. There exist $\phi^{ \pm} \in C^{1}$ so that

$$
\begin{aligned}
\left(d \circ \phi^{ \pm}\right)(z) & =t_{e}-(z-s)^{2}-z \text { for all } z \in Z, \\
\left(\phi^{ \pm}\right)^{\prime}(0) & =-\mu \phi^{ \pm}(0)+f\left(\phi^{ \pm}\left(\tau^{ \pm}(0)\right)\right) .
\end{aligned}
$$

Define $x^{ \pm}:\left[-h, t_{e}\right] \rightarrow \mathbb{R}$ by $x_{0}^{ \pm}=\phi^{ \pm}$and

$$
x^{ \pm}(t)=e^{-\mu t} \phi^{ \pm}(0)+\int_{0}^{t} e^{-\mu(t-u)} f\left(\phi^{ \pm}\left(\tau^{ \pm}(u)\right)\right) d u
$$

for $0<t \leq t_{e}$. The functions $x^{ \pm}$are continuously differentiable. For all $t \in\left[0, t_{e}\right]$ we have

$$
\begin{aligned}
\left(x^{ \pm}\right)^{\prime}(t) & =-\mu x^{ \pm}(t)+f\left(\phi^{ \pm}\left(\tau^{ \pm}(t)\right)\right) \\
& =-\mu x^{ \pm}(t)+f\left(x^{ \pm}\left(t+r^{ \pm}(t)\right)\right), \\
\Delta\left(r^{ \pm}(t), x_{t}^{ \pm}\right) & =d\left(x_{t}^{ \pm}\left(r^{ \pm}(t)\right)\right)+r^{ \pm}(t) \\
& =d\left(x^{ \pm}\left(t+r^{ \pm}(t)\right)\right)+\tau^{ \pm}(t)-t \\
& =d\left(\phi^{ \pm}\left(\tau^{ \pm}(t)\right)\right)+\tau^{ \pm}(t)-t \\
& =t_{e}-\left(\tau^{ \pm}(t)-s\right)^{2}-\tau^{ \pm}(t)+\tau^{ \pm}(t)-t \\
& =0, \\
\operatorname{det} D_{1} \Delta\left(r^{ \pm}(t), x_{t}^{ \pm}\right) & =d^{\prime}\left(x_{t}^{ \pm}\left(r^{ \pm}(t)\right)\right)\left(x_{t}^{ \pm}\right)^{\prime}\left(r^{ \pm}(t)\right)+1 \\
& =d^{\prime}\left(\phi^{ \pm}\left(\tau^{ \pm}(t)\right)\right)\left(x^{ \pm}\right)^{\prime}\left(t+r^{ \pm}(t)\right)+1 \\
& =d^{\prime}\left(\phi^{ \pm}\left(\tau^{ \pm}(t)\right)\right)\left(\phi^{ \pm}\right)^{\prime}\left(t+r^{ \pm}(t)\right)+1 \\
& =\left(d \circ \phi^{ \pm}\right)^{\prime}\left(\tau^{ \pm}(t)\right)+1 \\
& =-2\left(\tau^{ \pm}(t)-s\right)-1+1 \\
& = \pm 2 \sqrt{t_{e}-t .}
\end{aligned}
$$

The last term is nonzero for $0 \leq t<t_{e}$ and vanishes at $t=t_{e}$. For $t \nearrow t_{e}$,

$$
\left(r^{ \pm}(t), x_{t}^{ \pm}\right) \rightarrow\left(r^{ \pm}\left(t_{e}\right), x_{t_{e}}^{ \pm}\right)=\left(s-t_{e}, x_{t_{e}}^{ \pm}\right),
$$

with respect to the norm on $B$. Set $w=s-t_{e}$ and $\psi^{ \pm}=x_{t_{e}}^{ \pm}$. Then $(w, \psi) \notin M$ since det $D_{1} \Delta(w, \psi)=0$, and the remaining assertions become obvious, with $s^{-}=$ $r^{-}(0)=\tau^{-}(0)=s+\sqrt{t_{e}}$ and $s^{+}=r^{+}(0)=\tau^{+}(0)=s-\sqrt{t_{e}}$. 


\section{REFERENCES}

[1] Alt, W., Periodic solutions of some autonomous differential equations with variable time delay. In: Functional Differential Equations and Approximation of Fixed Points, Bonn 1978, pp. 16-31, Peitgen, H.O., and H.O. Walther eds., Lecture Notes in Math., vol. 730, Springer, Berlin 1979.

[2] Arino, O., Hadeler, K. P., and M. L. Hbid, Existence of periodic solutions for delay differential equations with state-dependent delay. J. Differential Eqs. 144 (1998), 263-301.

[3] Diekmann, O., van Gils, S. A., Verduyn Lunel, S. M., and H. O. Walther, Delay Equations: Functional-, Complex- and Nonlinear Analysis. Springer, New York, 1995.

[4] Foley, C., and M. C. Mackey, Dynamic hematological disease: A review. Preprint, 2007.

[5] Hale, J. K., and S. M. Verduyn Lunel, Introduction to Functional Differential Equations. Springer, New York 1993.

[6] Hartung, F., Krisztin, T., Walther, H. O., and J. Wu, Functional differential equations with state-dependent delay: Theory and applications. In HANDBOOK OF DIFFERENTIAL EQUATIONS, Ordinary Differential Equations, volume 3, pp. 435-545, Canada, A., Drabek., P. and A. Fonda eds., Elsevier Science B. V., North Holland, Amsterdam 2006.

[7] Libet, B., Wright, E. W., Feinstein, B., and D. Pearl, Subjective referral of the timing for a conscious sensory experience: A functional role for the somatosensory specific projection system in man. Brain 102 (1979), 193-224.

[8] Krisztin, T., $C^{1}$-smoothness of center manifolds for differential equations with statedependent delays. In Nonlinear Dynamics and Evolution Equations, Fields Institute Communications 48 (2006), 213-226.

[9] Krisztin, T., and O. Arino, The 2-dimensional attractor of a differential equation with state-dependent delay. J. Dynamics and Differential Eqs. 13 (2001), 453-522.

[10] Kuang, Y., and H. L. Smith, Periodic solutions of differential delay equations with thresholdtype delays. In Oscillation and Dynamics in Delay Equations, pp. 153-176, Graef, J. R., and J. K. Hale eds., Contemporary Mathematics, vol. 120, Amer. Math. Soc., Providence, 1992.

[11] Kuang, Y., and H. L. Smith, Slowly oscillating periodic solutions of autonomous statedependent delay differential equations. Nonlinear Analysis TMA 19 (1992), 855-872.

[12] Mackey, M. C., personal communication.

[13] Magal, P., and O. Arino, Existence of periodic solutions for a state-dependent delay differential equation. J. Differential Eqs. 165 (2000), 61-95.

[14] Mallet-Paret, J., and R. D. Nussbaum, Boundary layer phenomena for differential-delay equations with state-dependent time-lags: I. Archive for Rational Mechanics and Analysis 120 (1992), 99-146.

[15] Mallet-Paret, J., and R. D. Nussbaum, Boundary layer phenomena for differential-delay equations with state-dependent time-lags: II. J. für die reine und angewandte Mathematik 477 (1996), 129-197.

[16] Mallet-Paret, J., and R. D. Nussbaum, Eigenvalues for a class of homogeneous cone maps arising from max-plus operators. Discrete and Continuous Dynamical Systems 8 (2002), 519-562.

[17] Mallet-Paret, J., and R. D. Nussbaum, A basis theorem for a class of max-plus eigenproblems, J. Differential Eqs. 189 (2003), 616-639.

[18] Mallet-Paret, J., and R. D. Nussbaum, Boundary layer phenomena for differential-delay equations with state-dependent time-lags: III. J. Differential Eqs. 189 (2003), 640-692.

[19] Mallet-Paret, J., Nussbaum, R. D., and P. Paraskevopoulos, Periodic solutions for functional differential equations with multiple state-dependent time lags. Topological Methods in Nonlinear Analysis 3 (1994), 101-162.

[20] Nussbaum, R. D., Periodic solutions of some nonlinear autonomous functional differential equations. Annali di Matematica Pura ed Applicata IV Ser. 101 (1974), 263-306.

[21] Qesmi, R., and H. O. Walther, Center-stable manifolds for differential equations with statedependent delay, Discrete and Continuous Dynamical Systems, to appear.

[22] Stumpf, E., in preparation.

[23] Walther, H. O., The solution manifold and $C^{1}$-smoothness of solution operators for differential equations with state dependent delay. J. Differential Eqs. 195 (2003), 46-65.

[24] Walther, H. O., Smoothness properties of semiflows for differential equations with state dependent delay. Russian, in Proceedings of the International Conference on Differential and 
Functional Differential Equations, Moscow, 2002, vol. 1, pp. 40-55, Moscow State Aviation Institute (MAI), Moscow 2003. English version: Journal of the Mathematical Sciences 124 (2004), 5193-5207.

[25] Walther, H. O., Stable periodic motion of a system using echo for position control. J. Dynamics and Differential Eqs. 15 (2003), 143-223.

[26] Walther, H. O., On a model for soft landing with state-dependent delay. J. Dynamics and Differential Eqs. 19 (2007), 593-622.

[27] Walther, H. O., A periodic solution of a differential equation with state-dependent delay. J. Differential Eqs. 244 (2008), 1910-1945. 
Mathematisches Institut, Universität Giessen, Arndtstr. 2, D 35392 Giessen, Germany.

E-MAIL Hans-Otto.Walther@math.uni-giessen.de, TelePhone $++49-641-9932120$, FAX $++49-$ 641-9932029 